\title{
Recent advances in the application of the Schwinger multichannel method with pseudopotentials to electron-molecule collisions
}

\author{
Romarly F. da Costa ${ }^{1}$, Márcio T. do N. Varella ${ }^{2}$, Márcio H.F. Bettega ${ }^{3, a}$, and Marco A.P. Lima ${ }^{4}$ \\ ${ }^{1}$ Centro de Ciências Naturais e Humanas, Universidade Federal do ABC, 09210-170 Santo André, São Paulo, Brazil \\ 2 Instituto de Física, Universidade de São Paulo, Caixa Postal 66318, 05315-970 São Paulo, São Paulo, Brazil \\ 3 Departamento de Física, Universidade Federal do Paraná, Caixa Postal 19044, 81531-990 Curitiba, Paraná, Brazil \\ 4 Instituto de Física "Gleb Wataghin", Universidade Estadual de Campinas, 13083-859 Campinas, São Paulo, Brazil
}

Received 20 March 2015 / Received in final form 8 May 2015

Published online 18 June 2015

(c) The Author(s) 2015. This article is published with open access at Springerlink.com

\begin{abstract}
The Schwinger multichannel method [K. Takatsuka and V. McKoy, Phys. Rev. A 30, 1734 (1984)], which is based on the Schwinger variational principle for the scattering amplitude [J. Schwinger, Phys. Rev. 72, 742 (1947)], was designed to account for exchange, polarization and electronically multichannel coupling effects in the low-energy region of electron scattering from molecules with arbitrary geometry. The applications of the method became more ambitious with the availability of computer power combined with parallel processing, use of norm-conserving pseudopotentials and improvement of the description of target excited states (minimal orbital basis for single configuration interaction). The most recent applications involving 33 and 45 electronically open channels for phenol and ethylene molecules, represent good examples of the present status of the method. In this colloquium, we review the strategy and point out new directions to apply the method in its full extension.
\end{abstract}

\section{Introduction}

Modeling of discharge environments is in general a good motivation for experimental and theoretical activities for examining electron-molecule scattering processes. Some of the examples are: (1) planetary atmospheres bombarded by photons and charged particles $[1,2],(2)$ practical understanding of atmospheric reentry physics in space programs [3], (3) modeling of chemical plasmas for surface treatments [4-7], (4) DNA fragmentation induced by electron attachment (motivation for studying several biomolecules)[8], (5) nanomaterials fabrication using focused electron-beam-induced processing $[9,10]$ and more recently (6) pre-treatment (de-lignification) of biomass to obtain biofuels [11-13].

Studies of electron-molecule scattering resonances for free electrons can be useful to understand anion-molecule collisions or even for neutral molecular donors of electrons reacting with other molecules. In these cases, the electron is just dressed in a different way (wave package shaped by its carrier) but the underlying physical/chemical process is similar.

From the theoretical point of view it is a many-body problem that needs to be approximated in a realistic way, in order to describe or predict experimental results. Description of the target is the first challenge. A molecule is composed by $N_{n}$ nuclei and $N$ electrons. This group

\footnotetext{
${ }^{a}$ e-mail: bettega@fisica.ufpr.br
}

of particles are bound together and form a molecule that can rotate, vibrate and change its nuclei and electronic structure (or a combination of these "motions") as a result of its interaction with an electron. The time scale of these processes is $\tau_{\text {elect }} \ll \tau_{\text {vibr }} \ll \tau_{\text {rot }}$ and when compared with typical electron-molecule collision times, as show in Table 1 (adapted from [14]), allows for a separation of the electronic and the nuclear motion and gives rise to a fixed-nuclei approximation. By evaluating the cross sections for a frozen molecule in its lowest-energy geometry (neglecting rotation and vibration), it is possible to obtain cross sections for a molecular gas in accord with experiments as long as the scattering electrons are sufficiently fast and experience no delay due to resonances. Strategies of including nuclear motion through a combination of snap shots of different molecular geometries are very common. The bottom line is that the electronic dynamics represent a great challenge for most of the electron-molecule scattering situations. The main effects that should be accounted for are: (1) the static potential (potential scattering due to the nuclei and electronic molecular density), (2) the exchange potential (potential due to anti-symmetrization of the $(N+1)$-identical electrons wave function), and (3) the polarization potential (short and long range potentials due to electronic cloud deformation caused by the scattering electron presence), and by considering what is known as multichannel coupling (flux competition among all possible electronic 
Table 1. Typical collision time scale (seconds). Adapted from reference [14].

\begin{tabular}{lccc}
\hline & $\mathrm{N}_{2}$ & $\mathrm{CF}_{3} \mathrm{I}$ & Typical \\
\hline Collision $(1 \mathrm{eV})$ & $1 \times 10^{-16}$ & $4 \times 10^{-16}$ & $1 \times 10^{-16}$ \\
Collision $(10 \mathrm{eV})$ & $5 \times 10^{-17}$ & $1 \times 10^{-16}$ & $3 \times 10^{-17}$ \\
Collision $(30 \mathrm{eV})$ & $3 \times 10^{-17}$ & $7 \times 10^{-17}$ & $2 \times 10^{-17}$ \\
Electronic Exc. & $4 \times 10^{-17}$ & $1 \times 10^{-16}$ & $5 \times 10^{-17}$ \\
Rotation & $1 \times 10^{-12}$ & $1 \times 10^{-11}$ & $1 \times 10^{-12}$ \\
Vibration & $1 \times 10^{-15}$ & $5 \times 10^{-15}$ & $1 \times 10^{-14}$ \\
\hline
\end{tabular}

transitions, including ionization, with all being due to the electron impact).

The first group of theoretical methods to address electron-molecule scattering was based on single-particle potential scattering $[15,16]$. The electron is subjected to an average potential and the method only describes the elastic processes. This strategy along the years included optical potentials for full electronic inelasticity [17-19]. For electronic excitation, the initial strategy was to use wave functions obtained in the single-particle potential scattering to evaluate inelastic cross sections within the distorted wave approximation method [20]. This could give, at best, what one would obtain in a two channel closecoupling calculation ${ }^{1}$. In order to include the electronic multichannel coupling, using the many-particle character of the wave function, several initiatives took place. Among them, we find (1) close-coupling calculations [21], (2) the linear algebraic method [22], (3) the R-matrix technique [23], (4) the Schwinger multichannel method [24,25], and (5) the complex-Kohn variational method [26]. Here we will discuss the evolution of the SMC method towards the Schwinger multichannel method with pseudopotentials (SMCPP) and its most interesting applications.

Takatsuka and McKoy constructed the fully ab initio Schwinger multichannel method [24,25], including all the above mentioned effects, except the ionization channel, in a $(N+1)$-particle wave function description. In order to be applicable to polyatomic molecular targets with arbitrary geometries, the natural strategy was to use Cartesian Gaussian functions for the expansion of the scattering wave function. For this, they relied on the Schwinger method strategy that allows a $L^{2}$ type (square integrable) expansion and puts all exit channels in a $(N$ electrons bounded and one electron free) Green's function (expressed in a $V G_{P}^{(+)} V$ term better described below). The whole strategy was based on the fact that 2-electron integrals involving 4 Cartesian Gaussian functions or oneplane wave and 3 Cartesian Gaussian functions could be evaluated analytically [27-29]. As a result the variational method would be transformed in simple matrix multiplications (in the same spirit of bound state quantum chemistry computer codes) involving these integrals. In order to achieve this goal, the first version of the SMC method relied on a Cartesian Gaussian projector (approximation of the unity operator) insertion technique on the left and right side of $G_{P}^{(+)}$in the $V G_{P}^{(+)} V$ term. This strategy

1 For a close-coupling calculation, see, for example [21]. was not precise and convergence of the cross sections, even for the elastic channel, needed a large number of trials of Cartesian Gaussian functions. Historically, the evolution of the method went through the following steps: (1) Cartesian Gaussian insertion with the computer code running in central memory [30,31]; (2) numerical integration of the residue part of the $V G_{P}^{(+)} V$ term $^{2}$; (3) NASA's reorganization of the computer codes aiming at intense use of I/O (disk memory instead of central memory) [33,34]. From this point two different versions of the program evolved in different ways, the version at Caltech bet on: (4) parallelization of the computer codes [35]; while the version at the State University of Campinas (UNICAMP) returned to a central memory strategy and bet on: (5) the use of norm-conserving pseudopotentials (SMCPP) [36]; both versions relied on a (6) three-dimension integration of the $V G_{P}^{(+)} V$ term [37]. From this point, the Brazilian version that produced the results described in this paper evolved to: (7) single excitation configuration interaction for the target description of the excited states and a strategy of a minimal orbital basis for single configuration interaction (MOBSCI) [38] for choosing the appropriate and feasible level of multichannel coupling computation; and finally moved to (8) consolidation of the central memory strategy with parallelization of the computer codes with all properties cited above [39].

In what follows, we present, in some detail, the resulting SMCPP method for electron-molecule scattering, developed along the last years. We also present a summary of its most ambitious applications and point new directions to consolidate the multichannel convergence process in order to obtain reliable cross sections.

\section{Theory}

The Schwinger multichannel (SMC) method $[24,25]$ is a variational strategy that uses $(N+1)$-particle square integrable basis functions to obtain the scattering amplitude for electron-molecule collisions. It considers the fixed-nuclei approximation and includes important effects such as exchange, polarization, and electronic multichannel coupling. In the present implementation the SMC method does not describe ionization. The scattering equation was obtained by Takatsuka and McKoy [24,25] by mixing the integral and differential forms of the Schrödinger equation to obtain:

$$
A^{( \pm)}\left|\Psi_{\mathbf{k}_{i}}^{( \pm)}\right\rangle=V\left|S_{\mathbf{k}_{i}}\right\rangle
$$

where $A^{( \pm)}$is an operator that includes the free (electron plus target with $N$ electrons) Green's Function $G_{0}^{( \pm)}=$ $\left(1-H_{0} \pm i \epsilon\right)^{-1}$ projected in the electronic open channel

\footnotetext{
2 The strategy for numerical evaluation of the residue of the $V G_{P}^{(+)} V$ term was developed by Gibson, it was first applied for $\mathrm{e}^{-}-\mathrm{CH}_{4}$ scattering in [32].
} 
space. For the outgoing Green's function, we have

$$
A^{(+)}=\frac{\hat{H}}{N+1}-\frac{(\hat{H} P+P \hat{H})}{2}+\frac{(V P+P V)}{2}-V G_{P}^{(+)} V .
$$

In the expressions above, $P$ is a projector onto the $N_{\text {open }}$ energy-allowed target electronic channels, i.e.

$$
P=\sum_{\ell=1}^{N_{\text {open }}}\left|\Phi_{\ell}\right\rangle\left\langle\Phi_{\ell}\right|
$$

$G_{P}^{(+)}$is the free-particle Green's function projected onto the $P$ space, $V$ is the projectile-target interaction potential and $\hat{H}=E-H$ is the total energy (ground state energy plus kinetic energy of the incoming electron) minus the Hamiltonian of the $(N+1)$ electrons under the field of the fixed nuclei. The latter is given by $H=H_{0}+V$, where $H_{0}$ describes the non-interacting electron-molecule system and $S_{\mathbf{k}_{\ell}}$ is a solution of $H_{0}$, namely the product of a plane wave (projectile) and a target state $\Phi_{\ell}$. Later on, Lima and McKoy [40] showed that equations (1) and (2) could be obtained by simply enforcing the combination

$$
a P\left|\Psi_{\mathbf{k}_{i}}^{(+)}\right\rangle+(1-a P)\left|\Psi_{\mathbf{k}_{i}}^{(+)}\right\rangle=\left|\Psi_{\mathbf{k}_{i}}^{(+)}\right\rangle
$$

to be a solution of the Schrödinger equation

$$
\begin{aligned}
(E-H)\left|\Psi_{\mathbf{k}_{i}}^{(+)}\right\rangle=(E-H) & \left(a P\left|\Psi_{\mathbf{k}_{i}}^{(+)}\right\rangle\right. \\
& \left.+(1-a P)\left|\Psi_{\mathbf{k}_{i}}^{(+)}\right\rangle\right)=0
\end{aligned}
$$

and by replacing the first $P\left|\Psi_{\mathbf{k}_{i}}^{(+)}\right\rangle$term with the projected Lippmann-Schwinger equation, i.e.

$$
P\left|\Psi_{\mathbf{k}_{i}}^{(+)}\right\rangle=P\left|S_{\mathbf{k}_{i}}\right\rangle+P G_{0}^{(+)} V\left|\Psi_{\mathbf{k}_{i}}^{(+)}\right\rangle .
$$

This strategy gives

$$
A^{(+)}=\frac{\hat{H}}{a}-\frac{(\hat{H} P+P \hat{H})}{2}+\frac{(V P+P V)}{2}-V G_{P}^{(+)} V .
$$

The value $a=N+1$ leads to $A^{(+)}$of equation (2) and it was obtained by enforcing the matrix elements of the differential operator $\frac{\hat{H}}{N+1}+\frac{\hat{H} P+P \hat{H}}{2}$, between asymptotically non-zero wave functions, to vanish. In this case, or in the earlier demonstration by Takatsuka and McKoy [24,25], the form of the operator $A^{(+)}$is for general target wavefunctions and it remains the same either in a Hartree-Fock description or in cases where electronically correlated targets are necessary. Here we now make a review of the basic concepts of the method and introduce a more formal way of finding the value of $a$ in equation (4).

The scattering amplitude (in atomic units) for an electron arriving in a $\mathbf{k}_{i}$ plane wave, hitting a target in its ground state $\Phi_{i}(1, \ldots, N)$, exciting it to $\Phi_{f}(1, \ldots, N)$ and leaving the scattering region in a $\mathbf{k}_{f}^{\prime}$ plane wave, is given by

$$
f\left(\mathbf{k}_{f}^{\prime}, \mathbf{k}_{i}\right)=-4 \pi^{2}\left\langle S_{\mathbf{k}_{f}^{\prime}}|V| \Psi_{\mathbf{k}_{i}}^{(+)}\right\rangle .
$$

It is important to note that $\left|\Psi_{\mathbf{k}_{i}}^{(+)}\right\rangle$is anti-symmetric with respect to any exchange of two electrons but $S_{\mathbf{k}_{f}^{\prime}}$ is not, if the scattering particle is involved in the exchange. A convenient way of dealing with this asymmetry is to enforce the normalization of $S_{\mathbf{k}_{j}}$ to be $\left\langle S_{\mathbf{k}_{f}^{\prime}} \mid S_{\mathbf{k}_{i}}\right\rangle=\delta\left(\mathbf{k}_{f}^{\prime}-\mathbf{k}_{i}\right) \delta_{i f}$, with the normalization of $\left|\Psi_{\mathbf{k}_{j}}^{(+)}\right\rangle$being

$$
\left\langle\Psi_{\mathbf{k}_{f}^{\prime}}^{(+)} \mid \Psi_{\mathbf{k}_{i}}^{(+)}\right\rangle=(N+1) \delta\left(\mathbf{k}_{f}^{\prime}-\mathbf{k}_{i}\right) \delta_{i f}
$$

This normalization is convenient because it gives the following asymptotic condition

$$
\begin{aligned}
& \lim _{r \rightarrow \infty} \Psi_{\mathbf{k}_{i}}^{(+)}\left(\mathbf{r}_{1}, \ldots \mathbf{r}_{N}, \mathbf{r}\right)=\Phi_{i}\left(\mathbf{r}_{1}, \ldots \mathbf{r}_{N}\right) \frac{e^{i \mathbf{k}_{i} \cdot \mathbf{r}}}{(2 \pi)^{3 / 2}} \\
& +\sum_{f}^{N_{\text {open }}}\left(-4 \pi^{2}\right)\left\langle S_{\mathbf{k}_{f}^{\prime}}|V| \Psi_{\mathbf{k}_{i}}^{(+)}\right\rangle \Phi_{f}\left(\mathbf{r}_{1}, \ldots \mathbf{r}_{N}\right) \frac{e^{i k_{f}^{\prime} r}}{r}
\end{aligned}
$$

while, the normalization $\left\langle\bar{\Psi}_{\mathbf{k}_{f}^{\prime}}^{(+)} \mid \bar{\Psi}_{\mathbf{k}_{i}}^{(+)}\right\rangle=\delta\left(\mathbf{k}_{f}^{\prime}-\mathbf{k}_{i}\right) \delta_{i f}$, gives asymptotically

$$
\begin{gathered}
\lim _{r \rightarrow \infty} \bar{\Psi}_{\mathbf{k}_{i}}^{(+)}\left(\mathbf{r}_{1}, \ldots \mathbf{r}_{N}, \mathbf{r}\right)=\frac{1}{\sqrt{N+1}} \Phi_{i}\left(\mathbf{r}_{1}, \ldots \mathbf{r}_{N}\right) \frac{e^{i \mathbf{k}_{i} \cdot \mathbf{r}}}{(2 \pi)^{3 / 2}} \\
+\sum_{f}^{N_{\text {open }}}\left(-4 \pi^{2}\right)\left\langle S_{\mathbf{k}_{f}^{\prime}}|V| \bar{\Psi}_{\mathbf{k}_{i}}^{(+)}\right\rangle \Phi_{f}\left(\mathbf{r}_{1}, \ldots \mathbf{r}_{N}\right) \frac{e^{i k_{f}^{\prime} r}}{r} .
\end{gathered}
$$

Both amplitudes give the same cross sections, if we consider that $\bar{\Psi}_{\mathbf{k}_{i}}^{(+)}=\frac{\Psi_{\mathbf{k}_{i}}^{(+)}}{\sqrt{N+1}}$ and that the incident flux of equation (10) is $(N+1)$ times bigger than the incident flux of equation (11). With the convenient normalization (Eq. (9)) in mind, let us define an anti-symmetrization operator $\mathcal{A}$ such as

$$
\mathcal{A}\left\{\Phi_{i}(1, \ldots, N) \varphi_{j}(N+1)\right\}=\chi_{m},
$$

where $m=\{i, j\}$ is a combined index and $\chi_{m}$ has a similar normalization as given by equation (9). If $\Phi_{i}(1, \ldots, N)$ belongs to the $P$ projector and $\varphi_{j}$ is a genuine continuum function (orthogonal to all bound orbitals), it is clear that

$$
P \chi_{m}=\Phi_{i}(1, \ldots, N) \varphi_{j}(N+1) .
$$

This type of configuration $\left(\chi_{m}\right)$ given by equations (12) and (13) (an anti-symmetrized product of an energetically open target state with an orthogonal continuum orbital) defines the open-channel Feshbach projector $P_{\text {Fesch }}[41,42]$. All the other configurations, either involving energetically closed target states or those given by products of open target states with bound orbitals (not orthogonal to the target orbitals ${ }^{3}$ ), belong to the closedchannel Feshbach projector $Q_{\text {Fesch }}$. In the original demonstration of the method [24,25], although not using this

3 This kind of configuration was kidnamed by Barry Schneider as correlation terms. In $\mathrm{H}_{2}$, for instance, the $1 \sigma_{g} 1 \sigma_{u}^{2}$ configuration has its origin in the $b^{3} \Sigma_{u}$ state. See, for example [43]. 
terminology, the authors showed that

$$
P_{\text {Fesch }}\left[\frac{\hat{H}}{N+1}-\frac{(\hat{H} P+P \hat{H})}{2}\right] P_{\text {Fesch }}=0 .
$$

This is an important property to keep the $L^{2}$ expansion strategy for the $(N+1)$-particle wave function of the Schwinger method. All matrix elements of this operator

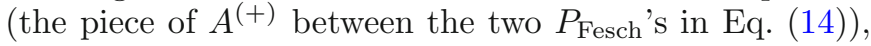
involving configurations of the $Q_{\text {Fesch }}$ space, can be evaluated using exclusively $L^{2}$ type of functions due to the short range nature of $Q_{\text {Fesch }}$ (ionization excluded). The matrix elements of all other operators in $A^{(+)}$(see Eq. (2)) contain $V$, and so they also can be evaluated exclusively with $L^{2}$ type of functions provided that $V$ vanishes faster than the Coulomb potential at infinity $(r \rightarrow \infty)$. Therefore, the condition given by equation (14) is sufficient to guarantee the desired property of expanding the scattering functions in square integrable functions.

In order to demonstrate equation (14) we first combine equations (12) and (13) to obtain

$$
\mathcal{A} P \chi_{\ell}=\chi_{\ell},
$$

and note that, with the normalization given by equation (9), it is simple to verify that

$$
\begin{array}{r}
\mathcal{A} \chi_{\ell}=(N+1) \chi_{\ell} \Rightarrow \mathcal{A}^{2}\left\{\Phi_{i}(1, \ldots, N) \varphi_{j}(N+1)\right\} \\
=(N+1) \mathcal{A}\left\{\Phi_{i}(1, \ldots, N) \varphi_{j}(N+1)\right\},
\end{array}
$$

or yet

$$
\mathcal{A}=\frac{1}{N+1} \mathcal{A}^{2}
$$

For any pair, $\chi_{m}$ and $\chi_{n}$, of configurations belonging to $P_{\text {Fesch }}$, we can write

$$
\begin{aligned}
\left\langle\chi_{m}|\hat{H} P| \chi_{n}\right\rangle & =\left\langle\mathcal{A} \Phi_{i} \varphi_{j}|\hat{H} P| \chi_{n}\right\rangle=\frac{\left\langle\mathcal{A}^{2} \Phi_{i} \varphi_{j}|\hat{H} P| \chi_{n}\right\rangle}{N+1} \\
& =\frac{\left\langle\mathcal{A} \Phi_{i} \varphi_{j}|\hat{H} \mathcal{A} P| \chi_{n}\right\rangle}{N+1}=\frac{\left\langle\chi_{m}|\hat{H}| \chi_{n}\right\rangle}{N+1},
\end{aligned}
$$

where we have used equations (15) and (17) and that $[\mathcal{A}, \hat{H}]=0$. An identical result can be obtained for the matrix element involving $P \hat{H}$, which combined with equation (18) proves equation (14). This result is general and independent of the level of correlation used for describing the target states $\Phi_{i}$.

A more straightforward way of obtaining a Schwinger multichannel equation was proposed by Germano and Lima [44] for positron-molecule scattering. With the help of the open $(P)$ and closed channel $(Q)$ projectors, where $P+Q=1$, the Schrödinger equation was written as

$$
(E-H)\left|\Psi_{\mathbf{k}_{i}}\right\rangle=(E-H)(P+Q)\left|\Psi_{\mathbf{k}_{i}}\right\rangle=0
$$

and the open channel part $P\left|\Psi_{\mathbf{k}_{i}}\right\rangle$ was again replaced by the projected Lippmann-Schwinger equation (Eq. (6)) to obtain

$$
A^{(+)}\left|\Psi_{\mathbf{k}_{i}}\right\rangle=V\left|S_{\mathbf{k}_{i}}\right\rangle \Rightarrow A^{(+)}=Q \hat{H} Q+P V P-V G_{P}^{(+)} V .
$$

The elegance of this operator comes from the fact that $Q \hat{H} Q$ and $P V P$ only couple closed- and open-space configurations, respectively, while $V G_{P}^{(+)} V$ couples the $P$ and $Q$ spaces. Why therefore can't we follow a similar elegant strategy for electron-molecule scattering? The difficulty lies in the definition of the $Q$ space. For any given target state belonging to $P$ space, which gives rise to an $(N+1)$-particle configuration through antisymmetrization (Eq. (12)), there exist $N$ other target states belonging to the $Q$ space giving rise to the same configuration. These $N$ states in $Q$ are constructed by exchanging the continuum state $\varphi$, associated to the scattering electron, with each one of the $N$ functions associated to the $N$ target electrons. This redundancy, indeed, guarantees that $(P+Q)=\mathbf{1}$ in the $(N+1)$-particle space, even if restricted to anti-symmetric states (i.e. $(P+Q)\left|\Psi_{\mathbf{k}_{i}}\right\rangle=$ $\left|\Psi_{\mathbf{k}_{i}}\right\rangle$ ). To construct $Q$, with this redundancy removed, we should first separate this specific set of continuum states ( $Q$ redundancy $)$ of the target and use it for defining a new projector $R=Q-Q_{\text {redundancy }}$. This can be very cumbersome. A better strategy is to note that the target states of $P+Q$ gives rise to $(N+1)$ times the number of linearly independent $(N+1)$-particle configurations that can be constructed with the help of equation (12). We, therefore, could make use of $\mathcal{A}(P+R)=\mathcal{A}(P+Q) /(N+1)=\frac{\mathcal{A}}{N+1}$ in equation (19) and in order to avoid constructing $R$, employ $\mathcal{A} R=\frac{\mathcal{A}}{N+1}-\mathcal{A} P$. Bringing $\mathcal{A}$ to the left of the Hamiltonian (they commute) in equation (19) and following the path described in reference [40], we obtain

$$
\mathcal{A}\left\{A^{( \pm)}\left|\Psi_{\mathbf{k}_{i}}^{( \pm)}\right\rangle-V\left|S_{\mathbf{k}_{i}}\right\rangle\right\}=0
$$

This is exactly the equation (1) obtained by the authors of the method $[24,25]$, except that it is multiplied by the antisymmetrizer $\mathcal{A}$. If we multiply this equation from the left by $\left\langle\Psi_{\mathbf{k}_{f}}^{(-)}\right|$, we get two of the usual definitions of the scattering amplitude, and the presence of $\mathcal{A}$ is just to remind us that the $(N+1)$-particle wave function must be antisymmetrized. This becomes even more evident in the bilinear form of the variational method for the scattering amplitude, given by

$$
\begin{aligned}
{[f]=} & -4 \pi^{2}\left\{\left\langle S_{\mathbf{k}_{f}^{\prime}}|V| \Psi_{\mathbf{k}_{i}}^{(+)}\right\rangle+\left\langle\Psi_{\mathbf{k}_{f}}^{(-)}|V| S_{\mathbf{k}_{i}}\right\rangle\right. \\
& \left.-\left\langle\Psi_{\mathbf{k}_{f}}^{(-)}\left|A^{(+)}\right| \Psi_{\mathbf{k}_{i}}^{(+)}\right\rangle\right\} .
\end{aligned}
$$

For independent variations of $\left|\delta \Psi_{\mathbf{k}_{i}}^{(+)}\right\rangle$and $\left\langle\delta \Psi_{\mathbf{k}_{f}}^{(-)}\right|$, we now get

$$
\begin{aligned}
& \left\langle S_{\mathbf{k}_{f}^{\prime}}|V| \delta \Psi_{\mathbf{k}_{i}}^{(+)}\right\rangle-\left\langle\Psi_{\mathbf{k}_{f}}^{(-)}\left|A^{(+)}\right| \delta \Psi_{\mathbf{k}_{i}}^{(+)}\right\rangle=0 \\
& \left\langle\delta \Psi_{\mathbf{k}_{f}}^{(-)}|V| S_{\mathbf{k}_{i}}\right\rangle-\left\langle\delta \Psi_{\mathbf{k}_{f}}^{(-)}\left|A^{(+)}\right| \Psi_{\mathbf{k}_{i}}^{(+)}\right\rangle=0 .
\end{aligned}
$$

This gives equation (21) (analogous to the original Eq. (1)) as long as the variations are arbitrary and on the antisymmetric space as it should be. This procedure makes clear that the $a=(N+1)$ factor in equation (4) is defined simply by the removal of the above mentioned exchange redundancy. It also shows that, as in the positron 
scattering case, the electron scattering $A^{(+)}$operator in equation (2) is obtained without specifying the level of approximation of the $N$-electron target and the $(N+1)$ particle scattering functions, i.e. $P$ can contain the number of open channels of choice and it can be built with or without target correlation.

\subsection{Implementation and numerical procedures}

Since the early 1990's, two implementations of the SMC approach were developed at Caltech $[45,46]$ and in Brazil, especially at UNICAMP. In the following, we briefly describe the key numerical aspects of the method and the main features of the Brazilian code, namely the use of pseudopotentials [36], the MOBSCI [38] approach to multichannel coupling, and the parallelization based on OpenMP directives [39].

\subsubsection{The scattering amplitude}

Once the essential properties of the $A^{(+)}$operator have been established, equation (1) can be viewed as an alternative form of the Lippmann-Schwinger equation, with the operator $A^{(+)}$given in equation (2) replacing the standard form $A^{(+)}=V-V G_{0}^{(+)} V$ [47], which is not suitable for numerical implementation $[24,25]$, where $G_{0}^{(+)}=$ $\left[E-H_{0}+i \eta\right]^{-1}$ is the free-particle Green's operator. Following a well-known procedure (see for instance Ref. [47]), equations (1) and (2) can be used to build up the bilinear form of the Schwinger variational principle, given above by equation (22). Then, by expanding the scattering state in a trial basis $\left\{\left|\chi_{m}\right\rangle\right\}$,

$$
\left|\Psi_{\mathbf{k}_{i}}^{(+)}\right\rangle=\sum_{m} c_{m}^{(+)}\left(\mathbf{k}_{i}\right)\left|\chi_{m}\right\rangle
$$

where $c_{m}^{(+)}\left(\mathbf{k}_{i}\right)$ is a variational coefficient, the working expression for the scattering amplitude can be obtained as (details given elsewhere [38])

$$
f\left(\mathbf{k}_{f}^{\prime}, \mathbf{k}_{i}\right)=-\frac{1}{2 \pi} \sum_{m, n}\left\langle S_{\mathbf{k}_{f}^{\prime}}|V| \chi_{m}\right\rangle\left(d^{-1}\right)_{m n}\left\langle\chi_{n}|V| S_{\mathbf{k}_{i}}\right\rangle,
$$

where now we consider the plane waves normalized as $8 \pi^{3} \delta\left(\mathbf{k}-\mathbf{k}^{\prime}\right)$ (note the factor $-1 / 2 \pi$ instead of $-4 \pi^{2}$ in the scattering amplitude). The $d_{m n}$ matrix element is given by:

$$
d_{m n}=\left\langle\chi_{m}\left|A^{(+)}\right| \chi_{n}\right\rangle
$$

with $A^{(+)}$given by equation (2).

The trial vectors $\left|\chi_{m}\right\rangle$ are $(N+1)$-particle configuration state functions (CSFs), built from spin-adapted, anti-symmetrized products of target electronic states and projectile scattering orbitals. The open electronic collision channels are included in the $P$ space and the dynamical response of the target electrons to the projectile field (correlation-polarization effects) is accounted for through virtual excitations of the target. In this case, the CSFs are given by

$$
\left|\chi_{m}\right\rangle=\mathcal{A}\left|\Phi_{i}(1, \ldots, N)\right\rangle \otimes\left|\varphi_{j}(N+1)\right\rangle,
$$

where for $i>0,\left|\Phi_{i}\right\rangle \equiv{ }^{(2 S+1)}\left(h_{i} \rightarrow p_{i}\right)$ is a singly excited state obtained by promoting one electron from a hole orbital $\left(h_{i}\right)$ of the ground state $\Phi_{0}(1, \ldots, N)$ to a particle orbital $\left(p_{i}\right)$, with either singlet $(S=0)$ or triplet $(S=1)$ spin coupling, though only $(N+1)$-electron configurations with total spin $S=1 / 2$ (doublets) are actually taken into account. If we have $N_{\text {open }}$ states in equation (3), this level of calculation is called an $N_{\text {open-channel coupling }}$ scheme at the static-exchange-plus-polarization (acronym is $N_{\text {open }}$ ch-sep) approximation.

Direct comparison between equations (25) and (8) gives the $L^{2}$ (short range) variational $(N+1)$-particle scattering state

$$
\left|\Psi_{\mathbf{k}_{i}}^{(+)}\right\rangle=\sum_{m, n}\left|\chi_{m}\right\rangle\left(d^{-1}\right)_{m n}\left\langle\chi_{n}|V| S_{\mathbf{k}_{i}}\right\rangle
$$

Comparison of equation (25) with another definition of the amplitude given by

$$
f\left(\mathbf{k}_{f}^{\prime}, \mathbf{k}_{i}\right)=-\frac{1}{2 \pi}\left\langle\Psi_{\mathbf{k}_{f}^{\prime}}^{(-)}|V| S_{\mathbf{k}_{i}}\right\rangle
$$

gives

$$
\left\langle\Psi_{\mathbf{k}_{f}^{\prime}}^{(-)}\right|=\sum_{m, n}\left\langle S_{\mathbf{k}_{f}^{\prime}}|V| \chi_{m}\right\rangle\left(d^{-1}\right)_{m n}\left\langle\chi_{n}\right| .
$$

It is important to note that the same scattering state, given by equation (28), is used to calculate the elastic and inelastic transitions by just using the appropriate $\left\langle S_{\mathbf{k}_{f}^{\prime}}\right|$ state in equation (8). The inelastic transition and elastic transition between excited states (including elastic, inelastic and super-elastic processes) can be obtained by the same scattering state, given by equation (30), using again the appropriate $\left|S_{\mathbf{k}_{i}}\right\rangle$. In other words, the variational calculation that generates these scattering states, $\left\langle\Psi_{\mathbf{k}_{f}^{\prime}}^{(-)}\right|$and $\left|\Psi_{\mathbf{k}_{i}}^{(+)}\right\rangle$, takes care of all the involved transitions at the same time. Another way of seeing this is by noting that once we have defined the $(N+1)$-particle trial basis set $\left\{\chi_{n}\right\}$ and the level of multichannel coupling through $P$ (where we have to choose the number and quality of target states), the operator

$$
F=\sum_{m, n} V\left|\chi_{m}\right\rangle\left(d^{-1}\right)_{m n}\left\langle\chi_{n}\right| V
$$

is completely defined and furnishes all the transitions between any two states $i$ and $j$ in the $P$ space through the direct evaluation of

$$
f\left(\mathbf{k}_{f}^{\prime}, \mathbf{k}_{i}\right)=-\frac{1}{2 \pi}\left\langle S_{\mathbf{k}_{f}^{\prime}}|F| S_{\mathbf{k}_{i}}\right\rangle .
$$

Considering the level of coupling among states, the different ranges of the potentials of the elastic transitions (either from the ground or from excited states), we need a balanced choice of the $(N+1)$-particle configuration space $\left\{\left|\chi_{m}\right\rangle\right\}$ and of the $P$ space for obtaining reliable elastic, inelastic and super-elastic cross sections with the Schwinger multichannel method. 


\subsubsection{Numerator and Green's operator matrix elements}

Most of the matrix elements comprised in the $d_{m n}$ matrix (Eq. (26)), often referred to as the denominator matrix elements, are similar to those present in bound-state electronic structure techniques, in particular HartreeFock and Configuration Interaction. Noteworthy exceptions are the numerator matrix elements $\left\langle S_{\mathbf{k}}|V| \chi_{m}\right\rangle$ in equation (25) and the projected Green's operator matrix elements in the configuration space $\left\langle\chi_{m}\left|V G_{P}^{(+)} V\right| \chi_{n}\right\rangle$ in equation (26), since these have no counterparts in standard Quantum Chemistry methods. At the atomicorbital level, the former requires the computation of twoelectron repulsion integrals involving three CGs and a plane wave [27-29],

$$
\int d^{3} r_{1} \int d^{3} r_{2} e^{-i \mathbf{k} \cdot \mathbf{r}_{1}} g_{\alpha}\left(\mathbf{r}_{1}\right) \frac{1}{r_{12}} g_{\beta}\left(\mathbf{r}_{2}\right) g_{\gamma}\left(\mathbf{r}_{2}\right),
$$

where $g_{\alpha}$ denotes a CG function. The computational effort scales as $\sim N_{g}^{3}$, where $N_{g}$ is the dimension of the CG set, and these integrals should be calculated for all scattering energies and directions (employing angular numerical quadratures in the latter case). In view of the very large number of these integrals, we routinely resort to a pseudopotential approach wherein the nucleus and core electrons of the atoms heavier than hydrogen are replaced by a pseudocore that produces the same field of the true atomic core in the valence region. The pseudopotential implementation of the SMCP method (SMCPP) is described shortly in Section 2.1.4.

The computation of the Green's operator matrix elements is a key aspect of the method as it accounts for the scattering boundary condition. The early algorithms $[24,25,30,31]$ were based on the insertion of numerically complete Gaussian basis sets on both sides of the $G_{P}^{(+)}$operator, but the slow convergence required very large sets. To overcome this problem, an alternative approach to the off-the-energy-shell integration in momentum space was proposed [37],

$$
\begin{aligned}
\left\langle\chi_{\mu}\left|V G_{P}^{(+)} V\right| \chi_{\nu}\right\rangle= & \sum_{m}^{\text {open }} P \int_{0}^{\infty} k^{2} d k \frac{g_{\mu \nu}^{m}(k)}{\frac{1}{2} k_{m}^{2}-\frac{1}{2} k^{2}} \\
& +\sum_{m}^{\text {open }} i \pi g_{\mu \nu}^{m}(k),
\end{aligned}
$$

where $P$ denotes the Cauchy's principal value, and

$$
g_{\mu \nu}^{m}(k)=\int d \hat{\mathbf{k}}\left\langle\chi_{\mu} \mid \Phi_{m} \mathbf{k}\right\rangle\left\langle\mathbf{k} \Phi_{m} \mid \chi_{\nu}\right\rangle .
$$

In this procedure, the radial $k$-space is discretized on the quadrature points $\left\{k_{j}\right\}$, where the $g_{\mu \nu}^{m}\left(k_{j}\right)$ matrix elements are calculated, and the $\left\{\hat{\mathbf{k}}_{l}\right\}$ angular quadratures are also employed to obtain the $g_{\mu \nu}^{m}$ elements, according to equation (35). While this numerical integration is significantly more demanding than the previous implementation based on a numerically complete single-particle space, it provides a faster convergence with respect to the Gaussian basis sets and proved to be more stable and reliable over a large number of applications.

\subsubsection{Multichannel coupling effects and the MOBSCI approach for defining the $\mathrm{P}$ space}

As mentioned before, the description of the many-body dynamics in electron-molecule collisions is (at least at the fixed-nuclei approximation) strongly affected by the inclusion and also by a proper balanced treatment of exchange, polarization and multichannel coupling effects. At sufficiently low impact energies, only the elastic channel is open (i.e., it is the only channel that is energetically accessible) and the collision process is dominated by the description of the distortion of the target's electronic cloud due to the presence of the incident electron, an effect which is taken into account by allowing virtual excitations (closed channel space) from the ground state, as explained in Section 2.1.1. This effect combined with the proper solution of the scattering problem, including the exchange interaction, is known as the static-exchangeplus-polarization (SEP) approximation. As the electron impact energy increases, several discrete and continuum states (above the ionization threshold) become energetically accessible giving rise to what is called the multichannel coupling effect.

The aspect that basically determines the level of multichannel coupling in a scattering calculation is the number of energy-allowed target electronic states entering the sum that appears in the projector operator $P$, as given in equation (3). Depending on the impact energy and on the thresholds of the electronic target states included in the expansion of the open channel space, a number that ranges from 1-channel up to $N_{\text {open-channels will be cou- }}$ pled and will compete among themselves for the flux that defines the cross section. In our strategy, the configuration space is kept the same for all $N_{\text {open }}$ ch-sep approximations (i.e., for calculations with $N_{\text {open }}=1,2,3, \ldots$ ), where more channels are open as the collision energy increases. Here it is worth noting that, from the point of view of the theoretical formalism of the SMCPP method, there is no restriction on the number of energy-allowed states that can be included in the $P$ operator (i.e., $N_{\text {open }}$ can be very large and, in principle, infinity). In practical applications, however, the active space of coupled states must be judiciously truncated due to the high cost of the scattering calculation when too big a value of $N_{\text {open }}$ is considered in equation (3). This happens because, for every electronic channel, a large numerical quadrature must be carried out in order to evaluate the projected Green's function of equation (2). From this, the first point that we must have in mind, when dealing with the multichannel coupling effect and analyzing its influence on the results, is: how many (and perhaps, which) electronic states must be included in a scattering calculation in order to provide cross sections in reasonable agreement with carefully conducted experiments, while still keeping the computational effort manageable.

Another aspect that contributes to the accuracy of the cross sections, obtained in a calculation carried out with the inclusion of the multichannel coupling effect, is related to the level of approximation used in the representation of the target states. This aspect is especially important 
since it defines the quality of the electronic states used in the expansion of the $P$ operator. To put this statement in a contextualized perspective, it is important to recall that in methods such as the R-matrix and the complexKohn, the description of the target electronic states allows the inclusion of correlation. On the other hand, until recently, the target electronic states in the SMCPP implementation were described in terms of the improved virtual orbital (IVO) approximation [48]. The use of IVOs in our computational codes provides a simple and good representation of the excited states in terms of one or two Slater determinants (depending on the desired spin coupling), but implicitly implies a serious restriction because, within this approximation, states with different spin coupling may be not equally well described. Indeed, singlet and triplet IVO states when simultaneously taken into account in the same close coupling calculation would be constructed from the same space orbital, and one of them would be poorly described. The $b^{3} \Sigma_{u}$ and $\mathrm{B}^{1} \Sigma_{u}$ states of the hydrogen molecule illustrate this difficulty: although they have very distinct characters (since they represent typical valence and Rydberg states, respectively), in the IVO approximation these states are constructed with the same $\sigma_{u}$ orbital. To work around this limitation, we have improved the description of the target in the computational codes of the SMCPP method by replacing the IVO approach with a single-excitation configuration interaction (SCI) treatment of the excited states. However, a side effect of using such a description is that all on-shell roots of the target Hamiltonian (physical and pseudo states lying below the scattering on-shell energy) must be considered as open channels. In other words, the use of a SCI representation for the target introduces a large family of pseudo states that must be included in the scattering calculation in order to properly account for the flux distribution among the participant channels. In a typical scattering calculation every channel brings complications since the cross sections are very sensitive to new thresholds and, just before a threshold, an upcoming channel can give rise to spurious structures (narrow bumps and troughs - that can become wide structures if you do not open a channel that should be open). Moreover, as pseudo states usually have inaccurate thresholds, lots of misplaced structures would deteriorate the quality of the integral cross sections (ICS) and, as a consequence, the energy dependence of a calculated ICS can become very different from what may be found in the experiment.

In summary, the intrinsic many-body character of the electron-molecule collision problem imposes the use of approximations, and this occurs for both bound state and scattering calculations. According to the above considerations, a good description of the target states added to the use of a strategy to minimize the open channel space represent crucial steps towards the choice for the level of multichannel coupling to be used in our calculations. Aiming to address these two questions together, we have recently developed the so called minimal orbital basis for single configuration interaction strategy [38]. Through the use of the this strategy, we constructed an open channel space, composed by a set of hole-particle orbitals, which is capable of reproducing the spectrum of excitation energies obtained with a full single configuration interaction (FSCI) calculation for energies below a previously defined cutoff energy value. At the same time, within this approach, the number of pseudo states is minimized and the open channel space becomes as compact as possible.

\subsubsection{Pseudopotential implementation in the SMC method}

The pseudopotentials of BHS [49] were obtained from allelectron atomic calculations carried out within the localdensity approximation. They have the property of normconservation introduced by Hamann et al. [50], which assures that the all-electron and pseudo-wave-function describing the valence electrons are identical beyond a core

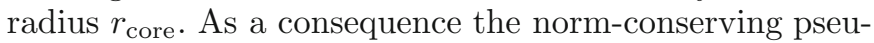
dopotentials are smooth near the origin, which makes easier any attempt to expand the wave function in a basis set. This is also an attractive property for scattering calculations, considering that the asymptotic region, where the pseudo and real wave functions are equal, governs the scattering properties.

The analytical expression for the BHS pseudopotentials, which are non-local, is

$$
\hat{V}_{\mathrm{PP}}=\hat{V}_{\text {core }}+\hat{V}_{\text {ion }}
$$

where

$$
\hat{V}_{\text {core }}=-\frac{Z_{\mathrm{val}}}{r} \sum_{i=1}^{2} c_{i} \operatorname{erf}\left(\rho_{i}^{1 / 2} r\right)
$$

where erf is the error function, and

$$
\hat{V}_{\text {ion }}=\sum_{n=0}^{1} \sum_{j=1}^{3} \sum_{\ell=0}^{2} A_{n j \ell} r^{2 n} e^{-\sigma_{j \ell} r^{2}} \sum_{m=-\ell}^{+\ell}|\ell m\rangle\langle\ell m| .
$$

$\hat{V}_{\text {core }}$ is finite at the origin and accounts for the valence Coulomb tail due to a nuclei with charge $Z_{\text {val }} \cdot \hat{V}_{\text {ion }}$ is short ranged and accounts for the ionic part. The set of parameters $A_{n j \ell}, \sigma_{j \ell}, c_{i}$ and $\rho_{i}$ are tabulated in the article of BHS [49].

In general, the pseudopotential implementation is done by replacing the nuclear potential $-Z / r$ by the pseudopotential operator $\hat{V}_{\mathrm{PP}}$. In the electronic-structure codes, the three-center integrals of the nuclear potential $-Z / r$ and two (real) atomic orbitals $\phi_{\mu}$ and $\phi_{\nu}$

$$
V_{\mu \nu}^{\mathrm{Nucl}}=\int d \boldsymbol{r} \phi_{\mu}\left[-\frac{Z}{r}\right] \phi_{\nu}
$$

are replaced by the three-center integrals of the pseudopotential $\hat{V}_{\text {PP }}$ and two atomic orbitals

$$
V_{\mu \nu}^{\mathrm{PP}}=\int d \boldsymbol{r} \phi_{\mu} \hat{V}_{\mathrm{PP}} \phi_{\nu} .
$$


In the scattering codes, the nuclear hybrid integrals of the nuclear potential and one atomic orbital $\phi_{\mu}$ and a plane wave $\phi_{\boldsymbol{k}}=e^{i \boldsymbol{k} \cdot \boldsymbol{r}}$ describing the continuum electron, as given by

$$
V_{\boldsymbol{k} \nu}^{\mathrm{Nucl}}=\int d \boldsymbol{r} \phi_{\boldsymbol{k}}^{*}\left[-\frac{Z}{r}\right] \phi_{\nu}
$$

are replaced by the nuclear hybrid integrals of the pseudopotential

$$
V_{\boldsymbol{k} \nu}^{\mathrm{PP}}=\int d \boldsymbol{r} \phi_{\boldsymbol{k}}^{*} \hat{V}_{\mathrm{PP}} \phi_{\nu}
$$

In our calculations, the atomic orbitals are represented by Cartesian Gaussians functions, given by

$g_{\alpha}^{\operatorname{lmn} \boldsymbol{A}}(\boldsymbol{r})=N_{l m n}\left(x-A_{x}\right)^{l}\left(y-A_{y}\right)^{m}\left(z-A_{z}\right)^{n} e^{-\alpha|\boldsymbol{r}-\boldsymbol{A}|^{2}}$.

With this choice the above integrals of the pseudopotential, as given by equations (36)-(38), are evaluated analytically. The Cartesian Gaussians functions are generated from atomic pseudo-wave-functions using a variational method, as described in reference [51]. This method employs a functional based on the Poisson equation, and provides Cartesian Gaussian functions which are obtained from the numerical atomic radial pseudo-wavefunctions. As we will show below, this method provides basis sets that are suitable for electronic elastic and inelastic calculations.

\subsubsection{Parallel implementation}

The computer code of the SMCPP method was recently redesigned [39] to improve the RAM memory efficiency and incorporate parallel processing. The parallelization was based on OpenMP directives (www.openmp.org) and significantly increased the performance in comparison with the previous serial version. For large target molecules, the number of numerator matrix elements $\left\langle S_{\mathbf{k}}|V| \chi_{m}\right\rangle$ in equation (25) is very large even with the use of pseudopotentials. The computation of these matrix elements and their transformation from the atomic-orbital to the molecular-orbital basis are now performed in parallel. The calculation of the $d_{m n}$ matrix of equation (26) and its inversion, according to equation (25), are also processed in parallel, where the last step can be performed with either the LU factorization or singular-value decomposition algorithms implemented in the linear algebra subroutines (BLAS) and LAPACK routines (www.netlib.org/lapack). The computation of the Green's operator elements and the off-shell integration (see Sect. 2.1.2) have also been parallelized, allowing for the use of either Gauss-Legendre or Lebedev-Laikov angular quadratures to perform the integration in equation (35). While the overall performance depends on the dimension of the GC and CSF bases, a typical speed up with 6 shared memory cores would be around a factor of 5 compared to a single core.

\subsection{Cross sections}

\subsubsection{Integral cross sections}

The integral cross section (ICS) for the electronic excitation process $\Phi_{n} \rightarrow \Phi_{n^{\prime}}$, where $\Phi_{n}$ denotes target states, can be readily obtained from the scattering amplitude given in equation (25),

$$
\sigma_{n \rightarrow n^{\prime}}(E)=\frac{k_{f}}{k_{i}} \frac{1}{4 \pi} \int d \hat{\mathbf{k}}_{i} \int d \hat{\mathbf{k}}_{f}\left|f\left(\mathbf{k}_{f}^{\prime}, \mathbf{k}_{i}\right)\right|^{2} .
$$

In the expression above, the magnitude of the final wave vector is given by $k_{f}^{2}=k_{i}^{2}-2\left(\epsilon_{n}^{\prime}-\epsilon_{n}\right)$, where $\epsilon_{n}$ denotes the energy of the $n$-th electronic target state. The integration over $\hat{\mathbf{k}}_{f}$ accounts for scattering into all the possible directions, while $(4 \pi)^{-1} \int d \hat{\mathbf{k}}_{i}$ averages over the random molecular orientations in the target gas. The integration over both the incoming and outgoing directions makes the ICS rotationally invariant and hence equal in both the molecule-fixed and laboratory-fixed reference frames defined below.

\subsubsection{Total cross sections}

The elastic amplitude contains information on both the elastic and inelastic transitions through the optical theorem. We can thus obtain the total cross section (TCS) from

$$
\sigma_{\text {tot }}=\frac{1}{4 \pi} \int d \hat{\mathbf{k}}_{i} \frac{4 \pi}{k} \operatorname{Im} f\left(\mathbf{k}_{i}, \mathbf{k}_{i}\right)=\sum_{n=1}^{N_{\text {open }}} \sigma(1 \rightarrow n),
$$

where $\sigma(1 \rightarrow n)$ is the integral cross section for the electronic transition $\Phi_{1} \rightarrow \Phi_{n}$. We have learned in recent applications that once the $(N+1)$-particle configuration space $\left\{\left|\chi_{n}\right\rangle\right\}$ is fixed, any change in $P$ affects the competition in a very interesting way among the open channels. The elastic cross sections, for instance, drops substantially as we open more and more channels, especially at intermediate energies $(<50 \mathrm{eV})$. This point will be reviewed later in the present paper.

\subsubsection{Partial waves and differential cross sections}

While the SMC method provides the scattering amplitude in the linear momentum representation, partial waves can be obtained from expansions of the ingoing and outgoing angular dependencies in terms of spherical harmonics $\left(Y_{\ell m}\right)[30,31]$. For instance, it is often useful to expand the outgoing wave vector in angular momentum components as follows,

$$
f\left(\mathbf{k}_{f}^{\prime}, \mathbf{k}_{i}\right) \equiv\left\langle\mathbf{k}_{f}^{\prime}|f| \mathbf{k}_{i}\right\rangle=\sum_{\ell=0}^{\ell_{\max }} \sum_{m=-\ell}^{\ell}\left\langle\hat{\mathbf{k}}_{f} \mid \ell m\right\rangle f_{\ell m}\left(k_{f}, \mathbf{k}_{i}\right),
$$


where $\left\langle\hat{\mathbf{k}}_{f} \mid \ell m\right\rangle=Y_{l m}\left(\hat{\mathbf{k}}_{f}\right)$ is a spherical harmonic and $\hat{\mathbf{k}}_{i}$ and $\hat{\mathbf{k}}_{f}$ are the incoming and outgoing directions in the molecule-fixed frame, where the scattering calculations are carried out (see below). The coefficient $f_{\ell m}\left(k_{f}, \mathbf{k}_{i}\right)=$ $\left\langle\ell m|f| \mathbf{k}_{i}\right\rangle$ can be understood as the scattering amplitude of an electron entering the interaction region in a planewave $\left|\mathbf{k}_{i}\right\rangle$ and leaving it in a partial wave $|\ell m\rangle$. Another way of calculating the scattering amplitude is by also expanding $\mathbf{k}_{i}$ in partial waves,

$$
\begin{aligned}
\left\langle\mathbf{k}_{f}^{\prime}|f| \mathbf{k}_{i}\right\rangle= & \sum_{\ell=0}^{\ell_{\max }} \sum_{m=-\ell}^{\ell} \sum_{\ell^{\prime}=0}^{\ell_{\max }} \sum_{m^{\prime}=-\ell^{\prime}}^{\ell^{\prime}}\left\langle\mathbf{k}_{f}^{\prime} \mid \ell m\right\rangle \\
& \times f_{\ell m, \ell^{\prime} m^{\prime}}\left(k_{f}, k_{i}\right)\left\langle\ell^{\prime} m^{\prime} \mid \mathbf{k}_{i}\right\rangle,
\end{aligned}
$$

where $f_{\ell m, \ell^{\prime} m^{\prime}}\left(k_{f}, k_{i}\right)=\left\langle\ell, m|f| \ell^{\prime}, m^{\prime}\right\rangle$ is the amplitude of an electron entering the interaction region in a partial wave $\left|\ell^{\prime}, m^{\prime}\right\rangle$ and leaving it in a partial wave $|\ell, m\rangle$.

To obtain the differential cross section (DCS), it is necessary to transform the scattering amplitude from the body-fixed (or molecule-fixed) reference frame (BF), where the calculations are most conveniently performed, to the laboratory-fixed frame (LF), where the experiments are carried out. The latter is defined such that its $z$-axis is aligned with the direction of the incident wave vector, i.e. $\mathbf{k}_{i}^{\prime}=k_{i} \hat{\mathbf{z}}^{\prime}$ (from now on we employ primed unit vectors to indicate directions in the LF). We thus use the outgoing angular momentum expansion, according to equation (46), and employ the well-known Wigner rotation matrices $\left(D_{m \mu}^{\ell}\right)[52]$ to obtain the scattering amplitude in the $\mathrm{LF}$,

$$
f^{\mathrm{LF}}\left(\mathbf{k}_{f}^{\prime}, \mathbf{k}_{i}\right)=\sum_{\ell=0}^{\ell_{\max }} \sum_{m=-\ell}^{\ell} f_{\ell m}^{\mathrm{LF}}\left(k_{f}, \mathbf{k}_{i}\right) Y_{\ell m}\left(\hat{\mathbf{k}}_{f}^{\prime}\right)
$$

with

$$
f_{\ell m}^{\mathrm{LF}}\left(k_{f}, \mathbf{k}_{i}\right)=\sum_{\mu=-\ell}^{\ell} D_{m \mu}^{*}(\Omega) Y_{\ell \mu}\left(\hat{k}_{f}^{\prime}\right)
$$

In the latter expression, $\Omega=(\alpha, \beta, \gamma)$ denotes the Euler angles [52] accounting for the $\mathrm{BF} \rightarrow \mathrm{LF}$ transformation. There is no need to transform the incoming angular dependence because the average over molecular orientations in the LF is equivalent to an average over incoming directions in the BF, such that $\hat{\mathbf{k}}_{i}$ can be related to the Euler angles. The DCS is then obtained from integrating over the azimuth angle $\left(\phi_{f}^{\prime}\right)$,

$$
\frac{d \sigma}{d \Omega}\left(\theta_{f}^{\prime}\right)=\frac{k_{f}}{k_{i}} \frac{1}{4 \pi} \int d \hat{k}_{i} \int_{0}^{2 \pi} d \phi_{f}^{\prime}\left|f^{\mathrm{LF}}\left(\boldsymbol{k}_{f}^{\prime}, \boldsymbol{k}_{i}^{\prime}\right)\right|^{2} .
$$

Finally, we mention that momentum transfer cross sections (MTCS) can be readily obtained from the DCS using,

$$
\sigma^{\mathrm{MT}}(E)=\int_{-1}^{1} d\left(\cos \theta_{f}^{\prime}\right)\left[1-\cos \theta_{f}^{\prime}\right] \frac{d \sigma}{d \Omega}\left(\theta_{f}^{\prime}\right) .
$$

\subsubsection{Born-closure corrections}

As pointed out in the previous Section, the finite basis sets employed in the SMC method effectively truncate the partial wave expansion of the scattering states. This is a significant limitation to describe scattering by polar targets since the dipole moment potential is very longranged, $V_{\text {dip }}=-\mathbf{D} \cdot \hat{\mathbf{r}} / r^{2}$, where $\mathbf{D}$ is the target permanent dipole moment. To improve the description of higher partial waves we routinely employ a Born-closure procedure [53], wherein the scattering amplitude for the dipole potential is obtained in closed form employing the first Born approximation (FBA). This simple approximation would be expected to properly describe the higher partial waves $(\ell \rightarrow \infty)$, such that we conveniently replace the lower angular momentum components as follows,

$$
\begin{aligned}
f_{\text {clos }}^{\mathrm{LF}}\left(\mathbf{k}_{f}^{\prime}, \mathbf{k}_{i}\right)= & f^{\mathrm{FBA}}\left(\mathbf{k}_{f}^{\prime}, \mathbf{k}_{i}\right)+\sum_{\ell=0}^{\ell_{\max }} \sum_{m=-\ell}^{\ell}\left[f_{\ell m}^{\mathrm{LF}}\left(k_{f}, \mathbf{k}_{i}\right)\right. \\
& \left.-f_{\ell m}^{\mathrm{FBA}}\left(k_{f}, \mathbf{k}_{i}\right)\right] Y_{\ell m}^{*}\left(\hat{\mathbf{k}}_{f}\right)
\end{aligned}
$$

In the above expression, $f^{\mathrm{FBA}}\left(\mathbf{k}_{f}^{\prime}, \mathbf{k}_{i}\right)$ is the FBA analytical scattering amplitude for the dipole potential obtained in the LF (the frame is indicated by the primed wave vector $\hat{\mathbf{k}}_{f}^{\prime}$, so we do not explicitly indicate the LF to simplify the notation). The amplitude $f_{\ell m}^{\mathrm{FBA}}\left(k_{f}, \mathbf{k}_{i}\right)$ is obtained from an outgoing partial wave expansion of the analytical FBA amplitude and $f_{\ell m}^{\mathrm{LF}}\left(k_{f}, \mathbf{k}_{i}\right)$ is given in equation (48).

While the DCS obtained from the Born-closure amplitude $\left(f_{\text {clos }}^{\mathrm{LF}}\right)$ are significantly improved at low scattering angles (below $\sim 30^{\circ}$ ), they are divergent in the very forward direction $\left(\theta_{f}^{\prime}=0\right)$ in elastic collisions, and hence do not allow for ICS computations. As discussed elsewhere [54], reasonable ICS estimates can be obtained by assuming rotationally inelastic collisions $\left(k_{i}^{2}=k_{f}^{2}+2 \Delta E_{\text {rot }}\right)$, since the condition $k_{f} \neq k_{i}$ removes the singularity. It is straightforward to show that this procedure is equivalent to obtaining approximate rotationally summed cross sections wherein all rotational excitations are assumed to have the same energy difference $\left(\Delta E_{\mathrm{rot}}\right)$, and the DCS is fairly insensitive to the choice of $\Delta E_{\text {rot }}$ beyond $\theta_{f}^{\prime}=1^{\circ}$ (the MTCS is even less sensitive due to the weighting factor $\left(1-\cos \theta_{f}^{\prime}\right)$ in the integrand of Eq. (51)). For high-symmetry target molecules $[55,56]$ an advantage may be taken from rotational selection rules, so this procedure can be stated on more rigorous grounds (see Sect. 2.2.5).

There is a very important aspect concerning the Born closure corrections to resonant elastic cross sections. The high partial waves accounted for by the $f^{\mathrm{FBA}}$ amplitude in equation (52) essentially impact the background, such that resonances are accounted for by the $f_{\ell m}^{\mathrm{LF}}$ amplitudes computed with the SMCPP method. For strongly polar targets the dipolar background can become large enough to obscure the resonance signatures $[54,57]$. In case the resonance spectrum is the problem of interest, we often avoid the Born-closure procedure and survey symmetry-resolved uncorrected cross sections that make the resonance states 
much more clear (at the expense of obtaining substantially underestimated cross section magnitudes).

The Born-closure procedure described here is also useful to improve the magnitude of electronically inelastic cross sections for spin-preserving dipole-allowed transitions. In these cases, the FBA scattering amplitude for the $1 \rightarrow n$ excitation is reasonably well described employing the dipole approximation,

$$
f^{\mathrm{FBA}}=-\frac{1}{2 \pi}\left\langle\mathbf{k}_{f} \Phi_{n}|V| \Phi_{1} \mathbf{k}_{i}\right\rangle \approx-\frac{1}{2 \pi}\left\langle\mathbf{k}_{f}\left|-\frac{\mathbf{d} \cdot \hat{\mathbf{r}}}{r^{2}}\right| \mathbf{k}_{i}\right\rangle,
$$

where $\mathbf{d}$ is the $1 \rightarrow n$ transition dipole moment. The above FBA amplitude can also be used in equation (52), such that the TCS in equation (45) can be alternatively obtained as

$$
\sigma_{\text {tot }}^{\text {clos }}=\sum_{n=1}^{N_{\text {open }}} \sigma^{\text {clos }}(1 \rightarrow n),
$$

where the rotationally inelastic approximation described above is applied to the elastic transition (polar targets only) and equation (53) is used to improve the electronically inelastic cross sections (spin-preserving dipoleallowed transitions only).

\subsubsection{Adiabatic excitation cross sections}

Even though the scattering calculations are carried out in the fixed-nuclei approximation, it is possible to obtain vibrational and rotational excitation cross sections, a posteriori, from the fixed-nuclei input. The simplest scheme to this end would be employing the adiabatic approximation [58], which assumes that the time scale for the excitation of some internal degree of freedom of the target is much larger than the collision time. In view of the very small energy spacings, rotational excitations are particularly suited for this approximation. The adiabatic scattering amplitude for the $0 \rightarrow \Gamma$ excitation, where $\Gamma$ denotes a complete set of rotational numbers, is given by

$$
f_{0 \rightarrow \Gamma}^{\mathrm{ADB}}\left(\mathbf{k}_{f}^{\prime}, \mathbf{k}_{i}\right)=-\frac{1}{2 \pi}\left\langle\Psi_{\Gamma}\left|f\left(\mathbf{k}_{f}^{\prime}, \mathbf{k}_{i}\right)\right| \Psi_{0}\right\rangle,
$$

where $\left|\Psi_{\Gamma}\right\rangle$ is a target rotational state and $f\left(\mathbf{k}_{f}^{\prime}, \mathbf{k}_{i}\right)$ is a fixed-nuclei elastic scattering amplitude (which is parametrically dependent on the target orientation in the LF).

A very useful relation between the elastic DCS and the rotationally summed DCS (RSDCS) can be easily derived by assuming that the molecule is in the rotational ground state and averaging the elastic cross section over the target orientations $\left(\Omega_{T}\right)$,

$$
{\frac{d \sigma^{\mathrm{vvr}}}{d \Omega}}^{\mathrm{a}}\left(\theta_{f}^{\prime}\right)=\int d \Omega_{T}\left|\Psi_{0}\left(\Omega_{T}\right)\right|^{2} \frac{d \sigma}{d \Omega}\left(\theta_{f}^{\prime} ; \Omega_{T}\right),
$$

where the parametric dependence on the target orientation is indicated by the semicolon. It is thus straightforward to use the completeness of the rotational eigenstates to obtain the rotationally summed (RS) cross section,

$$
\frac{d \sigma}{d \Omega}^{\mathrm{avr}}\left(\theta_{f}^{\prime}\right) \equiv \frac{d \sigma}{d \Omega}^{\mathrm{RS}}\left(\theta_{f}^{\prime}\right)=\sum_{\Gamma=0}^{\infty} \frac{d \sigma}{d \Omega}(0 \rightarrow \Gamma),
$$

where the $0 \rightarrow \Gamma$ rotational excitation DCS is indicated (this result assumes the validity of the adiabatic approximation and negligible rotational excitation energies with respect to the collision energy [55]). The fact that molecules are rotationally hot in gas-phase experiments is not important since the RSDCS should be understood as an approximation to the elastic DCS. As a consequence, we can profit in two different ways from the adiabatic approximation for rotational excitations. First, equation (55) provides a means to estimate the rotational excitation cross sections (for any $\Gamma \rightarrow \Gamma^{\prime}$ transition, not only from the ground state). Second, the RSDCS in equation (57) is particularly useful for highly symmetric targets $[55,56]$ that impose stringent selection rules for dipole-allowed $0 \rightarrow \Gamma$ rotational excitations. As a result, the Born-closure procedure (Eq. (52)) can be applied only to the dipole-allowed rotational excitations. Since these are often inelastic, the divergence in the forward direction is more rigorously removed, and the elastic DCS can be obtained as

$$
\frac{d \sigma}{d \Omega}=\sum_{\Gamma=0}^{\infty}{\frac{d \sigma^{\text {clos }}}{d \Omega}}^{(0 \rightarrow \Gamma)}
$$

with Born-closure corrections applied only to the dipoleallowed transitions.

\section{Results and discussion}

\subsection{Elastic scattering: single open channel}

In this section we present our results for elastic scattering in the static-exchange (SE) and in the static-exchange plus polarization (SEP) approximations. In all calculations we consider only the elastic channel opened.

The first applications of the SMCPP method considered elastic scattering by small molecules, such as $\mathrm{CH}_{4}$, $\mathrm{SiH}_{4}$, and $\mathrm{GeH}_{4}[36]$. After testing the pseudopotential implementation in these molecules, we investigated electron collisions with molecules containing heavier atoms, such as $\mathrm{CF}_{4}, \mathrm{CCl}_{4}, \mathrm{SiF}_{4}, \mathrm{SiBr}_{4}$ and $\mathrm{SiI}_{4}[59,60]$.

To illustrate the usefulness of pseudopotentials in the scattering calculations, we shown in Figures 1-4 differential cross sections for electron collisions with $\mathrm{CF}_{3} \mathrm{Br}$ and $\mathrm{CF}_{3} \mathrm{I}$ molecules obtained in the static-exchange approximation [61]. With the use of pseudopotentials, bromine and iodine are treated at the same level, since they have the same number of valence electrons. We also present experimental data from references [62-66]. In general, our results agree well with the experimental data for energies below $20 \mathrm{eV}$. At higher energies, our calculations lie above the experiment. This is understood in terms of the electronic inelastic channels that are closed, although they should be open at those energies. As a consequence, there is no flux loss from the elastic into the inelastic ones. As we will show below, the opening of the inelastic channels influences the elastic one, lowering the magnitude of the elastic cross sections. It is worth to mention that by 


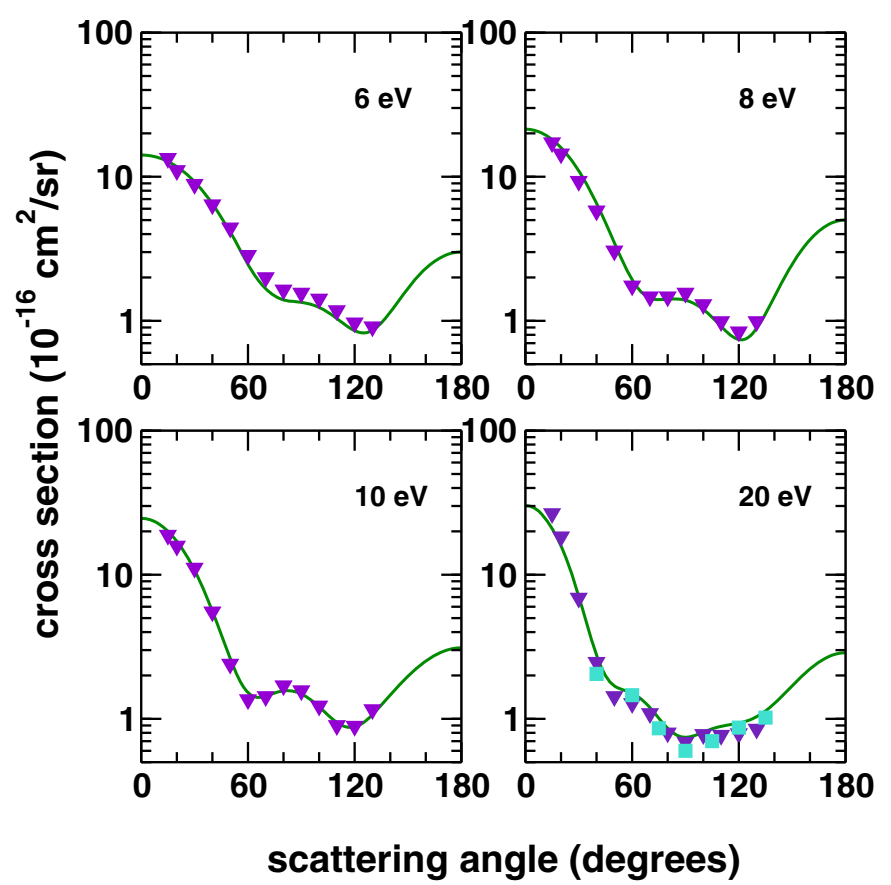

Fig. 1. Differential cross sections for electron scattering by $\mathrm{CF}_{3} \mathrm{Br}$ at $6,8,10$ and $20 \mathrm{eV}$. Solid line, theoretical results from reference [61] obtained in the static-exchange approximation; triangles, experimental data from reference [62]; squares, experimental data from reference [63]. Adapted from reference [61].

construction, the pseudopotentials of BHS include scalar relativistic effects, which are important for heavier atoms.

Figure 5 shows the integral cross sections for $\mathrm{SiY}_{4}(\mathrm{Y}=\mathrm{Cl}, \mathrm{Br}, \mathrm{I})$, obtained in the static-exchangepolarization approximation, from reference [67]. These results also illustrate the importance of the pseudopotentials in this type of calculations. The measured total cross sections from references $[68,69]$ are shown for comparison. Our results agree well with the experiment in shape and also in the location of the shape resonance spectra, where the computed resonance positions agree quite well with the experimental positions.

Motivated by experimental works on electron collisions with isomers of $\mathrm{C}_{3} \mathrm{H}_{4}$ and $\mathrm{C}_{4} \mathrm{H}_{6}$ [70-73], our group carried out a series of calculations in order to discuss the isomer (or isomeric) effect. The isomer effect allows one to distinguish between different isomers of a given molecule by differences in the scattering cross sections (total, integral elastic, differentials, rotation excitation). We investigated isomers of the hydrocarbons $\mathrm{C}_{3} \mathrm{H}_{4}, \mathrm{C}_{4} \mathrm{H}_{6}, \mathrm{C}_{4} \mathrm{H}_{8}$, $\mathrm{C}_{4} \mathrm{H}_{10}$ and $\mathrm{C}_{3} \mathrm{H}_{6}$ [74-79]. The results for energies between $10 \mathrm{eV}$ and $40 \mathrm{eV}$, suggested that the integral cross sections for the isomeric group differed by a factor that could be obtained through a scaling law based on geometrical optics (in fact this is a general model that was applied for molecules other than hydrocarbons) [80].

More recently, in collaboration with Vincent McKoy and Carl Winstead, from Caltech, and with Murtadha A. Khakoo, from CSFU, we investigated electron collisions

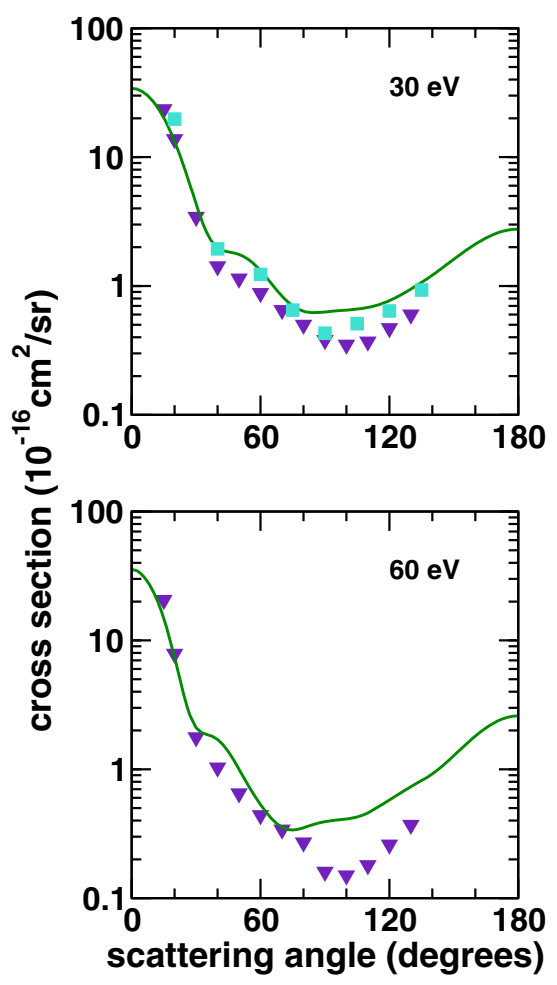

Fig. 2. Differential cross sections for electron scattering by $\mathrm{CF}_{3} \mathrm{Br}$ at 30 and $60 \mathrm{eV}$. Solid line, theoretical results obtained in the static-exchange approximation; triangles, experimental data from reference [62]; squares, experimental data from reference [63].

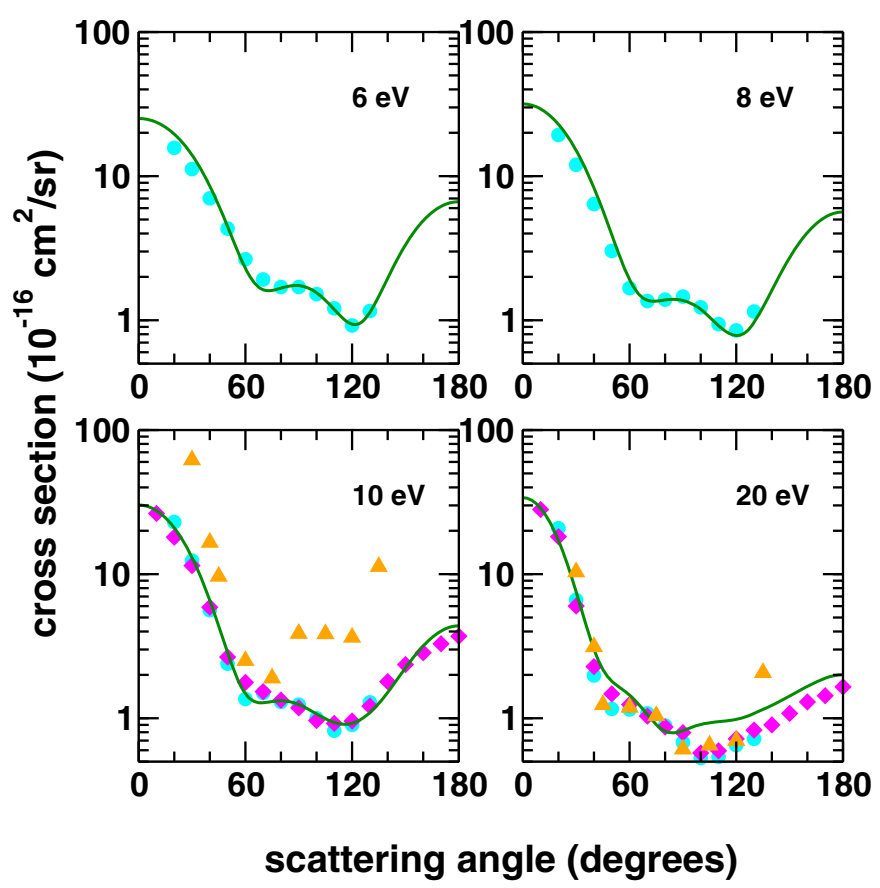

Fig. 3. Differential cross sections for electron scattering by $\mathrm{CF}_{3} \mathrm{I}$ at $6,8,10$ and $20 \mathrm{eV}$. Solid line, theoretical results from reference [61] in the static-exchange approximation; circles, experimental data from reference [64]; diamonds, experimental data from reference [65]; triangles, experimental data from reference [66]. Adapted from reference [61]. 


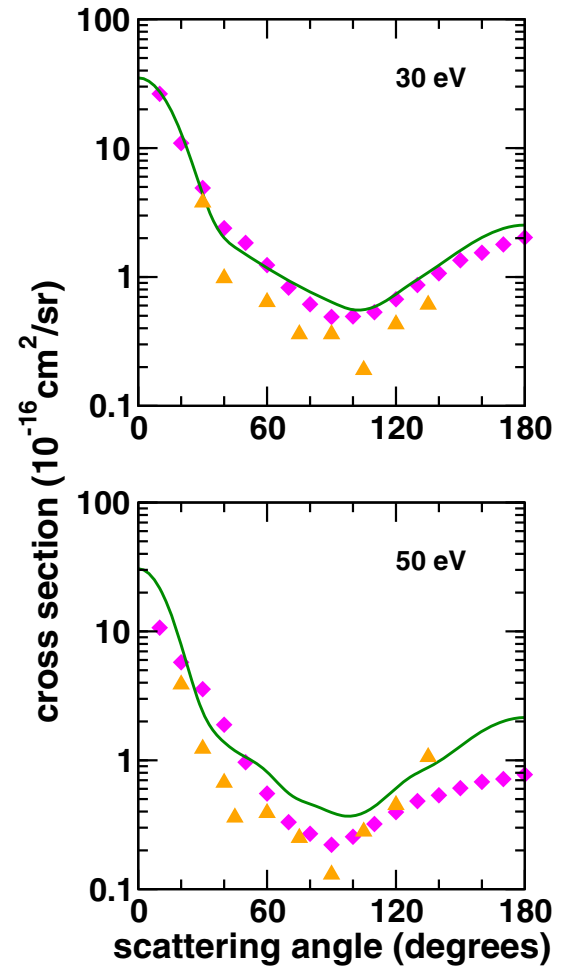

Fig. 4. Differential cross sections for electron scattering by $\mathrm{CF}_{3} \mathrm{I}$ at 30 and $50 \mathrm{eV}$. Solid line, theoretical results in the static-exchange approximation; diamonds, experimental data from reference [65]; triangles, experimental data from reference [66].

with alcohols, and discussed the behavior of the DCSs in terms of the molecular chain: for isomers (of alcohols and related alkanes and amines) with a straight chain, the DCSs display a characteristic $f$-wave behavior around the energy of the broad resonance which is present in the (elastic or total) cross sections, while isomers with a branched chain show a characteristic $d$-wave behavior [81-84].

The formic acid molecule can be considered a "toy model", before studying more complex molecules of biological relevance, since it has a characterized $\pi^{*}$ shape resonance located at around $1.9 \mathrm{eV}$ [85-92]. In Figure 6 we show our computed differential cross section at $135^{\circ}$ as a function of the impact energy, compared to the complex-Kohn results [90] and to the experimental results of Allan [91]. The two theoretical methods locate the resonance at the experimental location of $1.9 \mathrm{eV}$. The fact that both theories provide the resonance thinner in width and higher in magnitude than the experiment is explained in terms of the fixed-nuclei approximation employed by both methods, which fails in the case of resonant scattering.

In DNA, the bases are bounded by two or three hydrogen bonds, depending on the nature of the bases. In order to explore the effect of the hydrogen bonds in the shape resonance spectra, we investigated the elastic scattering of electrons by two dimers, a formic acid dimer (FAD) and a formamide dimer (FD), and the formic acidformamide complex (FAFC) [93]. All these systems contain two hydrogen bonds. Formamide also presents a well
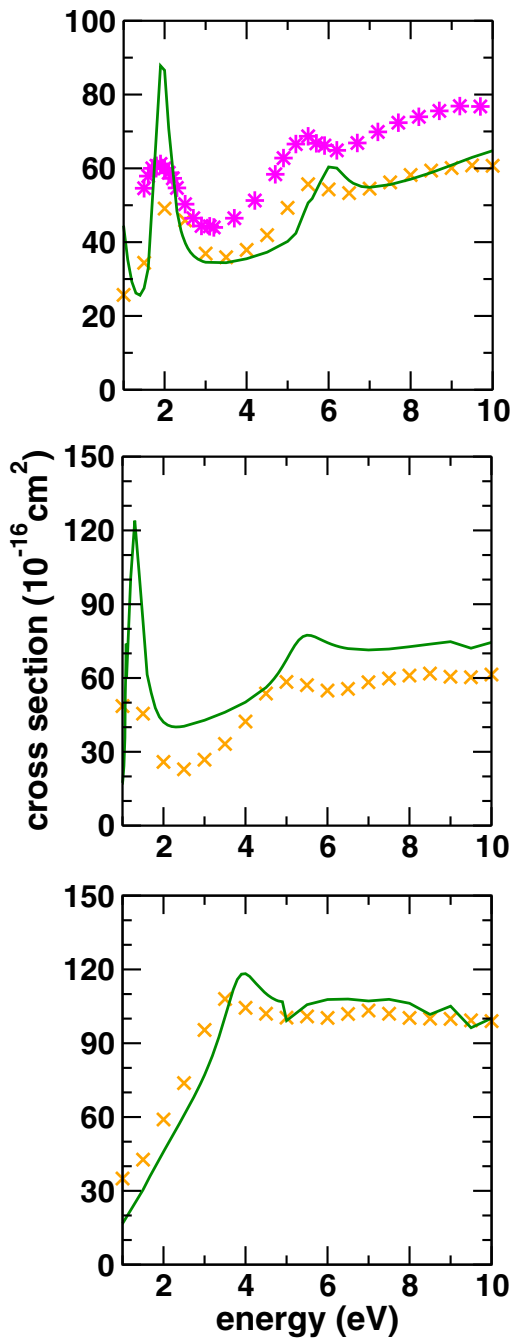

Fig. 5. Integral cross sections for electron collisions with $\mathrm{SiCl}_{4}$ (top), $\mathrm{SiBr}_{4}$ (middle) and $\mathrm{SiI}_{4}$ (bottom). We also present the measured total cross sections from reference [68] (crosses) and from reference [69] (stars). Adapted from reference [67].

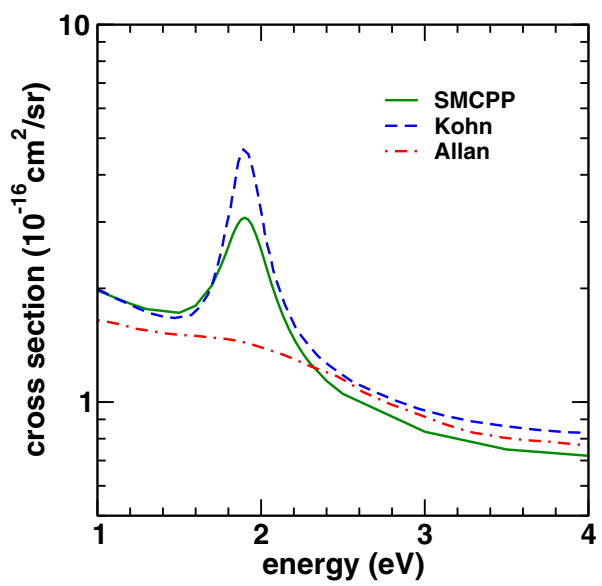

Fig. 6. Differential cross section for electron collisions with formic acid at a scattered angle of $135^{\circ}$ as a function of the energy. Solid line, present results; dashed line, results of the complex-Kohn method [90]; dot-dashed line, experimental data of Allan [91]. 

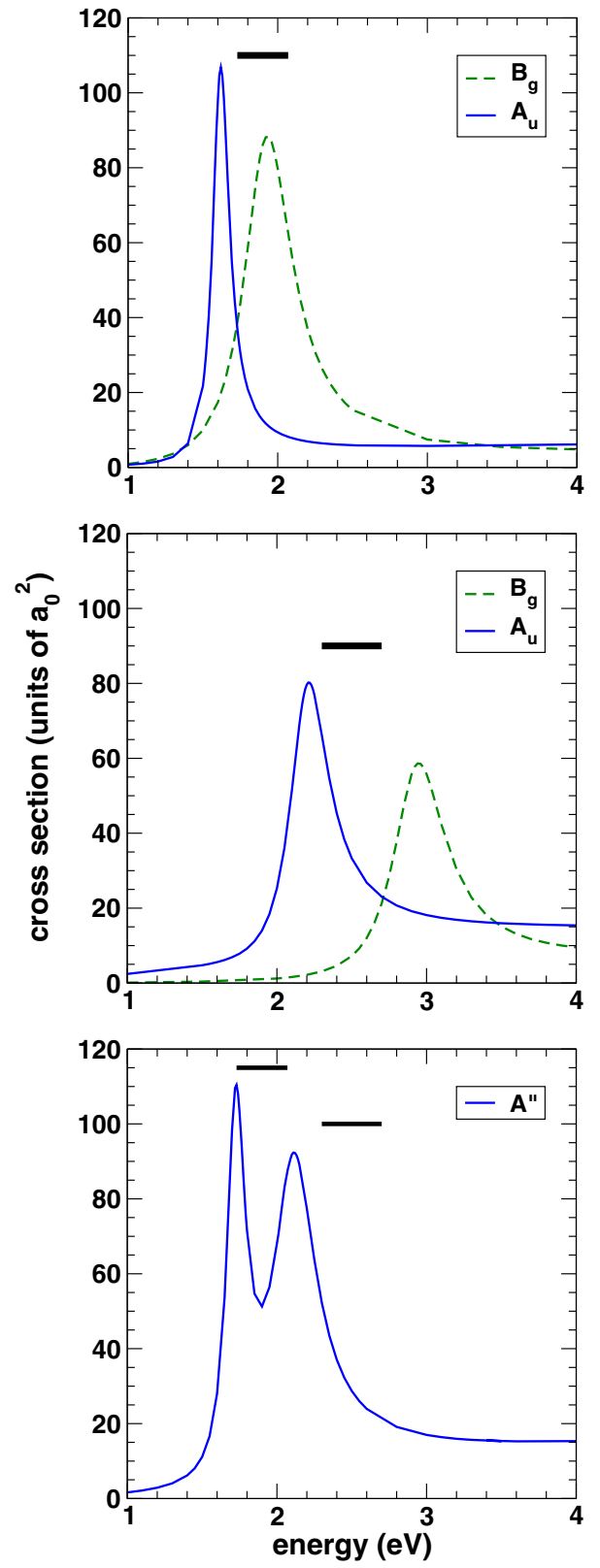

Fig. 7. Integral cross sections for the resonant symmetries of FAD, FD and FAFC. The bars indicate the resonances of $\mathrm{HCOOH}$ and of $\mathrm{HCONH}_{2}$ in the gas phase, which are centered at $1.9 \mathrm{eV}$ and $2.5 \mathrm{eV}$ respectively. The bars width represents the width of the resonances estimated from the calculations.

characterized $\pi^{*}$ shape resonance at around $2 \mathrm{eV}$ [94-98], which also allows the investigation of the formation of the resonances in the dimers and in the complex from the resonances of the units. Figure 7 shows the integral cross sections for the resonant symmetries of the FAD, FD and FAFC. Each system presents two shape resonances. The bars indicate the resonance of the units, which are centered at the calculated positions $(1.9 \mathrm{eV}$ for formic acid [92] and $2.5 \mathrm{eV}$ for formamide [98]) and the width is also estimated from the calculations. In the case of the FAD and FD the $A_{u}$ shape resonance is located below the resonance energy of the corresponding unit and the $B_{g}$ resonance is located above the resonance of the corresponding unit. For the FAFC the resonances stabilize with respect to the resonances of the units.

Figure 8 illustrates the formation of the resonances in the FAD (the same discussion holds for FD) and FAFC from the resonance of the units in a simple molecular orbital model. For the FAD, which has a $D_{2 h}$ symmetry, the LUMO ( $a_{u}$ symmetry) and the LUMO+1 ( $b_{g}$ symmetry) are located on the units and are even ("ungerade" with respect to the symmetry center) and odd ("gerade" with respect to the symmetry center) combinations of the LUMOs ( $a^{\prime \prime}$ symmetry) of the units. In the case of the FAFC, the LUMO and LUMO+1 (both of $a^{\prime \prime}$ symmetry) are located on the corresponding unit.

Another problem that we were able to study was the influence of microsolvation in the shape resonance of small molecules such as formaldehyde [99], formic acid [100] and phenol [101]. We show in Figures 9-11 the cross sections for complexes of formic acid with one and two surrounding water molecules. These studies represent the first step towards more complicated problems involving the effects produced by solvation in the resonance spectra of more complex molecules. It is important to remember, however, that doing experiments on electron scattering with solvated molecules is very difficulty, and theoretical efforts must start simple in order to learn and control the approximations employed in the calculations.

In the complexes $\mathbf{A}$ to $\mathbf{F}$, the water molecule plays the role of proton donor in the hydrogen bond, and the resonance of the complexes stabilize with respect to the resonance of formic acid in gas phase. The same occurs for the complexes $\mathbf{K}$ to $\mathbf{M}$, where the two water molecules are proton donors in the hydrogen bonds. The opposite occurs when the water molecules play the role of proton acceptors. For the complexes $\mathbf{G}$ to $\mathbf{J}$, where the water molecule is the proton acceptor, the resonance of the complexes destabilize with respect to the resonance in gas phase. In the complexes $\mathbf{N}$ to $\mathbf{P}$, one molecule is a proton donor and the other is a proton acceptor, and the resonance of the complexes also destabilizes. These results can be understood in terms of the net charge in the solute, where the resonant orbital is more concentrated. The resonance stabilization occurs when the net charge is positive, which means that the continuum electrons feels a more attractive potential than in the gas phase. The resonance destabilization corresponds to a net negative charge in the solute.

Furan, pyrrole and thiophene, along with their aza derivatives, can be considered prototypes of DNA/RNA basis and other molecules of biological relevance. For example, pyrrole is an analogue of one of the rings of adenine. The knowledge of the shape resonance spectra of these molecules can provide some insight to people investigating DEA to DNA. We obtained the elastic cross sections for these molecules [102-105] and also investigated electron collisions with their aza derivatives [106] namely, pyrazole, imidazole, isoxazole, oxazole, isothiazole and thiazole. We present in Table 2 our results for the position of the two $\pi^{*}$ shape resonances of furan, pyrrole and thiophene in 

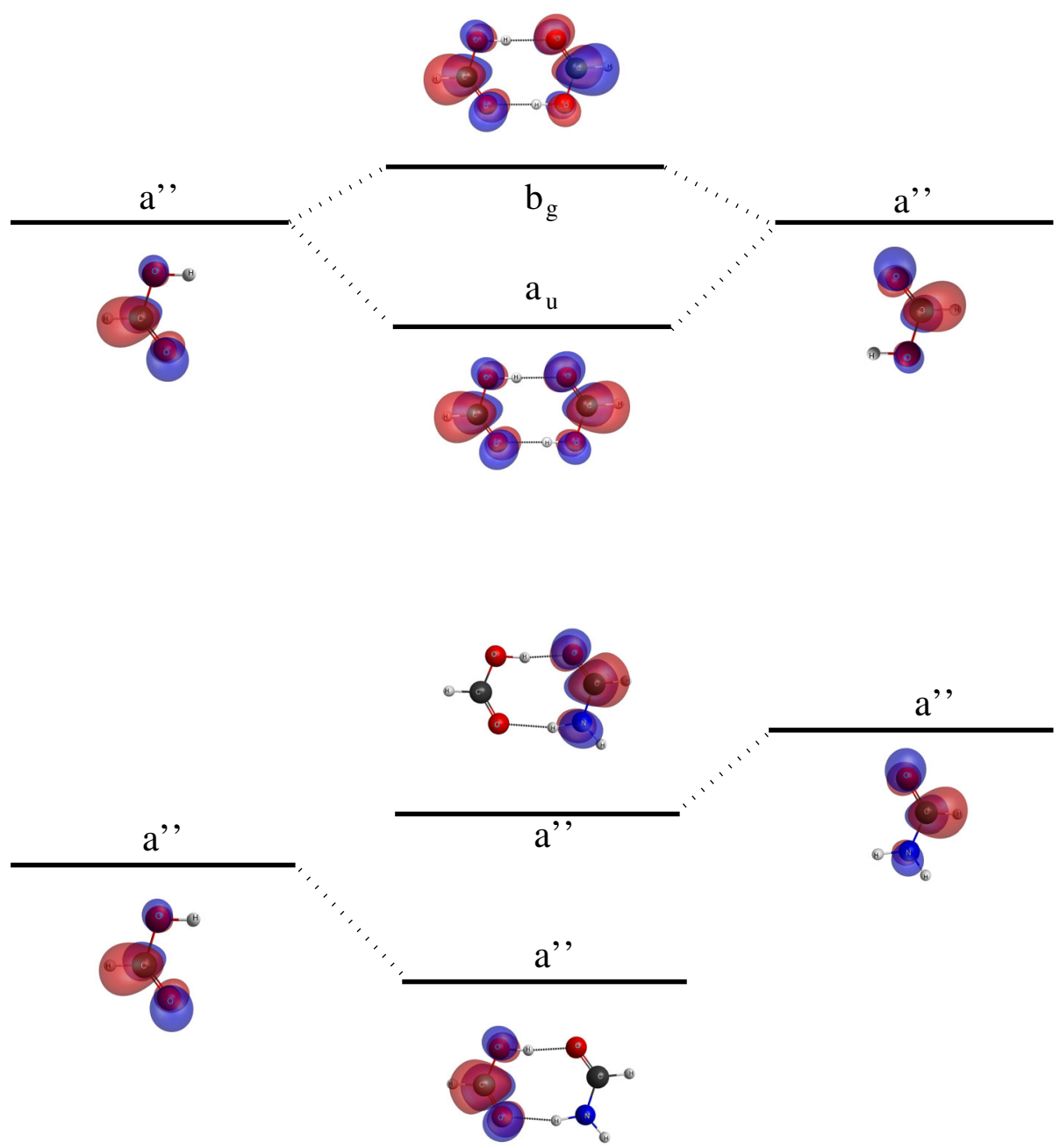

Fig. 8. Model based on the molecular orbital theory showing a pictorial view of the formation of the shape resonances in the FAD, FD and FAFC from the units formic acid and formamide.

comparison with the experimental data of Modelli and Burrow [107]. In general, our calculations reproduce well the experimental data. The same level of agreement was found among the calculated positions for the two $\pi^{*}$ shape resonances of the aza derivatives, as shown in Table 3 . We present in Figure 12 the cross sections for the $A^{\prime \prime}$ symmetry of the six aza derivatives. The bars are centered at the experimental value of the resonance, and the widths were estimated from the computed cross sections.

As mentioned before, the main motivation to study electron interactions with biomolecules is related to the radiation damage to DNA [108], i.e., the dissociation processes induced by secondary electrons released by the primary interaction of high-energy radiation with the biological matter. While these reactions triggered off by electron attachment are generally detrimental, as they can result in single and double DNA strand breaks, restricting their effect to cancer cells can actually be beneficial. In fact, it is possible to enhance the damage to tumor cells by incorporating radiosensitising drugs to the gene sequence through chemotherapy. Since halopyrimidines are found among the chemicals with potential radiosensitising ac- tivity $[109,110]$, we have recently addressed 2-chloro, 2bromo, and 5-bromopyrimidine [111], and uracil (U), 5fluorouracil (FU) and 5-chlorouracil (ClU) in a scattering study [57] (results for 5-bromouracil and 5-iodouracil [112] are not shown here). The use of pseudopotentials in combination with parallel processing is particularly important for these systems.

$\mathrm{U}$ and the halouracils have complex anion state spectra comprising dipole bound states (DBSs), valence bound states and shape resonances (in addition to the coreexcited states not addressed in the single-channel scattering study). In each case, the DBS was obtained with basis sets generated according to Skurski et al. [113] and incorporating coupled-cluster $(\mathrm{CCSD}(\mathrm{T}))$ perturbative corrections, as shown in Table 4. For all systems, we obtained three $\pi^{*}$ anion states, as shown in Figure 13 and also in Table 4 . The lowest-lying $\left(\pi_{1}^{*}\right)$ is a valence bound state in $\mathrm{ClU}$ and a shape resonance in U. For FU, the electron transmission (ET) spectrum [114] indicates an extremely low-lying $\pi_{1}^{*}$ resonance around $0.17 \mathrm{eV}$, while the SMCPP calculations comprising $4998 \mathrm{CSFs}$ ( $A^{\prime \prime}$ symmetry) predict a shallow bound state, $-0.09 \mathrm{eV}$ (the binding energies 

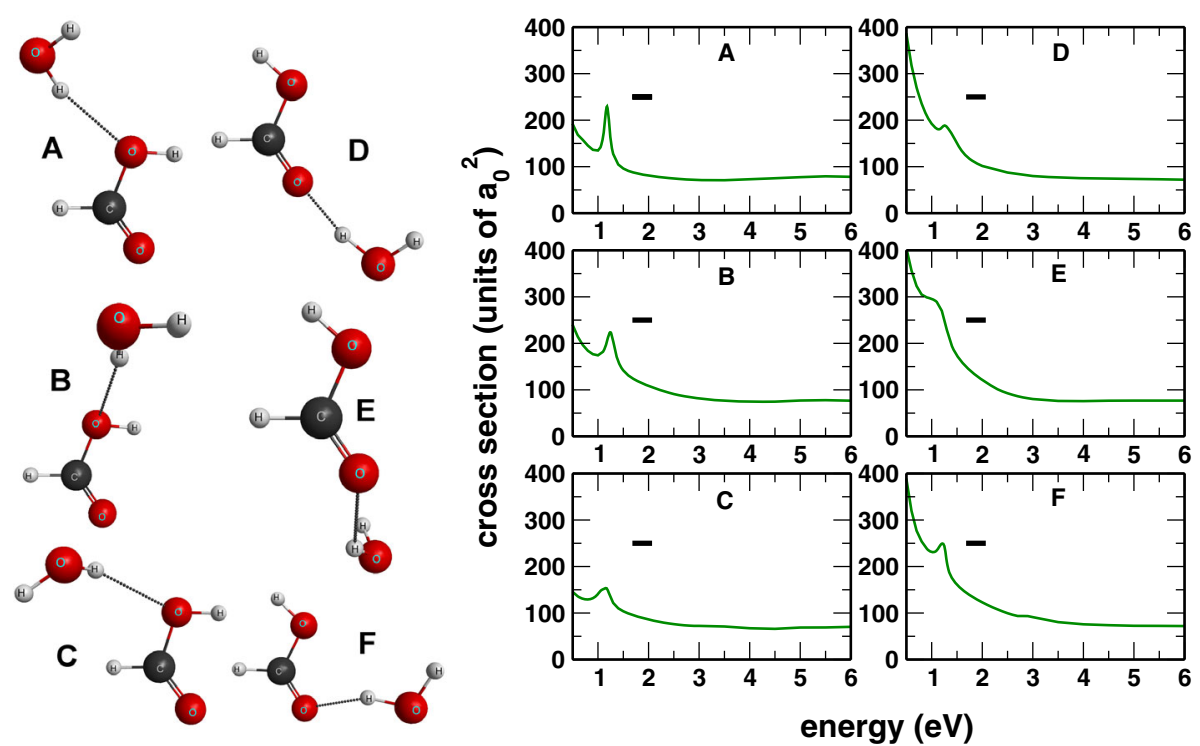

Fig. 9. Left: structure of the complexes from A to F. Right: momentum transfer cross sections in the SEP approximation for the six complexes $\mathbf{A}$ to $\mathbf{F}$. The bars indicate the resonance of $\mathrm{HCOOH}$ in the gas phase, which is centered at $1.9 \mathrm{eV}$. The bars width represents the width of the resonance estimated from the calculations. Adapted from reference [100].
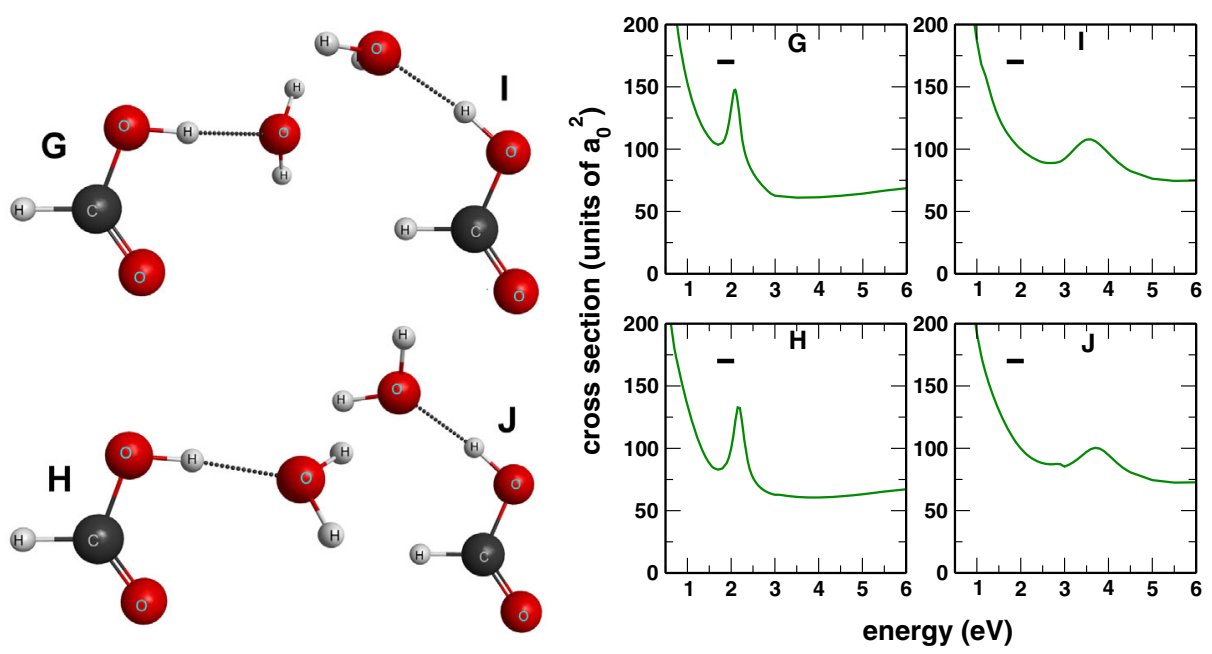

Fig. 10. Same as in Figure 9 for the four complexes G to J. Adapted from reference [100].

were obtained from the diagonalization of the scattering Hamiltonian represented in the CSF basis, and they are indicated as negative values). With 2001 CSFs, we obtained a shape resonance $(+0.09 \mathrm{eV})$, in qualitative agreement with the ET data, but we observe that both results are in good agreement $(<0.3 \mathrm{eV})$ with the experimental result for $\mathrm{FU}$. The $\pi_{2}^{*}$ and $\sigma_{\mathrm{CCl}}^{*}$ resonances are also consistent with the ET data, even though the $\pi_{3}^{*}$ resonance energies are overestimated due to the neglect of multichannel couplings in the elastic calculations. For comparison, we also show in Figure 13 the cross sections for 2-thiouracil (2$\mathrm{S}-\mathrm{U})$, another drug with radiosensitising potential [116]. The low-energy anion spectrum is similar to those of the halouracils, comprising a DBS $(-64 \mathrm{meV})$, three $\pi^{*}$ anion states at $-0.22 \mathrm{eV}, 0.56 \mathrm{eV}$ and $4.9 \mathrm{eV}$, according to the SEP calculation, in addition to a $\sigma^{*}$ resonance around $3.2 \mathrm{eV}$. The $\pi_{1}^{*}, \pi_{2}^{*}$ and the $\sigma^{*}$ anion states have significant probabilities on the $\mathrm{C}=\mathrm{S}$ group. The latter re- sults are also consistent with the recent calculations for dimethyl-disulfide [117], a prototype system to model the sulfide bridges that take part in the stabilization of the secondary structure of proteins. In this case, the S-S bond gives rise to a resonance with a strongly repulsive potential surface, which in turn leads to the cleavage within the Franck-Condon region of the S-S stretch mode of the neutral species.

\subsection{Applications of the SMC for open-shell molecules}

Over the years we had very few applications of the SMC method to study electron scattering from open-shell molecular targets. Open shell molecules are usually very reactive and some can be found as neutral radicals produced in discharge environments. The most famous openshell molecule is $\mathrm{O}_{2}$, followed by $\mathrm{NO}$ that can be formed in 


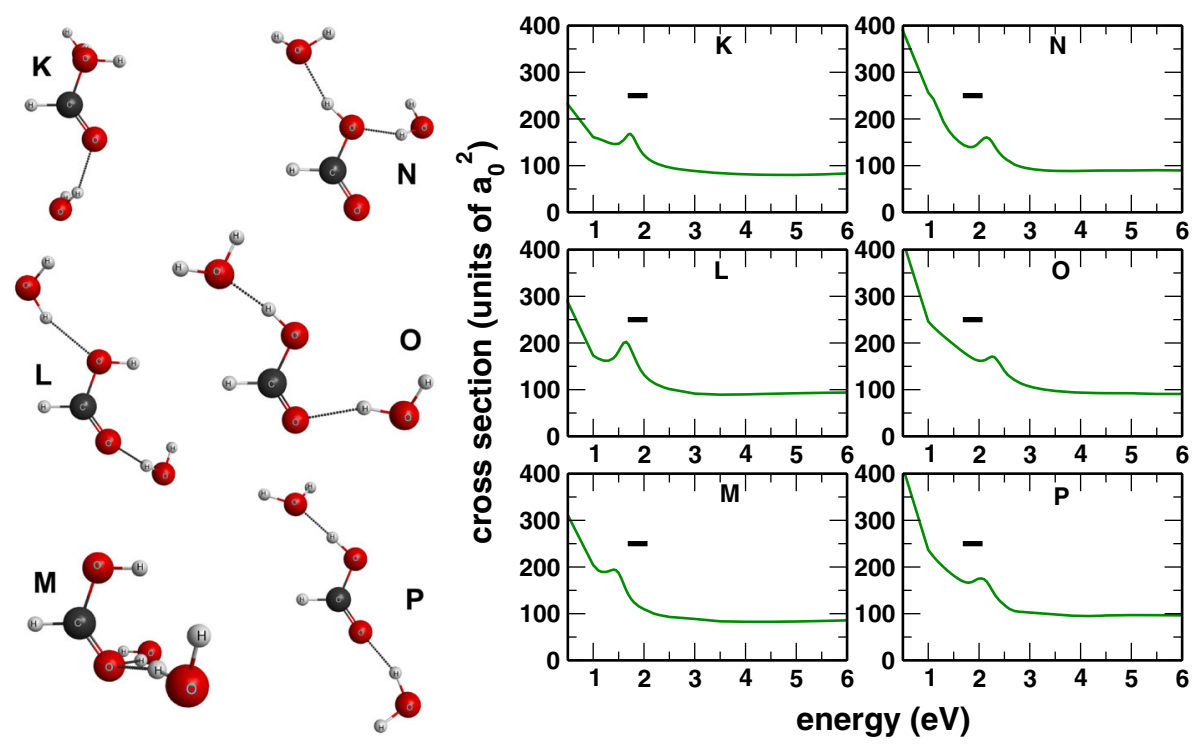

Fig. 11. Same as in Figure 10 for the six complexes $\mathbf{K}$ to $\mathbf{P}$. Adapted from reference [100].

Table 2. Measured vertical attachment energies (VAEs) from [107], and the calculated $\pi^{*}$ resonances positions with the SMCPP (SEP) method for furan [103], pyrrole [104] and thiophene [105]. All values are in eV. Adapted from reference [105].

\begin{tabular}{lcccc}
\hline & \multicolumn{2}{c}{$\pi^{*}\left(b_{1}\right)$} & \multicolumn{2}{c}{$\pi^{*}\left(a_{2}\right)$} \\
\hline & $\mathrm{VAE}_{\text {expt. }}$ & SMCPP & VAE $_{\text {expt. }}$ & SMCPP \\
\hline Furan & 1.73 & 1.95 & 3.15 & 3.56 \\
Pyrrole & 2.36 & 2.70 & 3.45 & 3.80 \\
Thiophene & 1.15 & 1.00 & 2.63 & 2.82 \\
\hline
\end{tabular}

Table 3. Calculated $\pi^{*}$ resonance positions with the SMCPP (SEP) method [106] and the measured vertical attachment energies (VAEs) from [107], for the aza-derivatives of furan, pyrrole and thiophene. All values are in $\mathrm{eV}$. Adapted from reference [106].

\begin{tabular}{lcccc}
\hline & \multicolumn{2}{c}{$\pi_{1}^{*}$} & \multicolumn{2}{c}{$\pi_{2}^{*}$} \\
\hline & SMCPP & VAE $_{\text {expt. }}$ & SMCPP & VAE $_{\text {expt. }}$ \\
\hline Pyrazole & 1.98 & 1.89 & 3.61 & 3.15 \\
Imidazole & 2.33 & 2.12 & 3.36 & 3.12 \\
Isoxazole & 0.87 & 1.09 & 3.26 & 2.77 \\
Oxazole & 1.53 & 1.44 & 3.14 & 2.81 \\
Isothiazole & 0.35 & 0.63 & 2.81 & 2.43 \\
Thiazole & 0.71 & 0.80 & 2.56 & 2.27 \\
\hline
\end{tabular}

a turbulent atmosphere of $\mathrm{N}_{2}$ and $\mathrm{O}_{2}$. A very interesting feature of electron collisions against an open-shell target, is that it allows the spin flip of the scattering electron even at zero impact energy. Hegeman et al. [118] have shown that in atomic target gases $(\mathrm{Na}$ and $\mathrm{Hg}$ ), the spin flip differential cross sections vary strongly with respect to scattering angle while for molecules like $\mathrm{O}_{2}$ and $\mathrm{NO}$ they were relatively smooth. In our first application [119] of the SMC method for electron scattering from the open-shell $\mathrm{O}_{2}$, we confirmed the experimental data and showed a strong dependence of the DCS with respect molecular orientation and that the interference pattern (so common in atomic cases) was washed out by the molecular orientational av- eraging. For this application, we have not designed a new computer program specifically to account for the spin coupling of a triplet ground state. Rather we used a complex Cartesian Gaussian version of our programs, and considered the initial state as a closed shell ${ }^{1} \Delta_{g}$ state and have used equation (31) to obtain all transitions among the $a^{1} \Delta_{g}, b^{1} \Sigma_{g}$, and $\mathrm{X}^{3} \Sigma_{g}^{-}$states of $\mathrm{O}_{2}$.

Following this application, the superelastic and electronic transitions out of excited states of $\mathrm{H}_{2}[120,121]$ were calculated with our regular computer codes (with the assumption of a Hartree-Fock closed shell molecule acting as the reference state). We have obtained superelastic cross sections as large as the elastic ground to ground state cross sections. We have also found elastic cross section between excited states almost an order of magnitude larger than the ground to ground state cross sections. The most important consequence of such large cross sections could be seen in a $\mathrm{H}_{2}$ plasma simulation carried out by Amorim et al. [122]. In that paper, a substantial change in the electron energy density distribution was obtained in a simulation of a discharge environment of $\mathrm{H}_{2}$ molecules, if the superelastic processes were included in the model. This change in electron energy density distribution can profoundly modify the chemistry of a plasma, and it should represent a good motivation for theoretical and experimental efforts to obtain cross sections from excited metastable states.

Nevertheless for addressing open-shell molecules, we have calculated cross sections for molecules containing one unpaired electron, like NO. To do so we had to develop a new set of computer codes that were specially designed for this purpose. Due to limited possibilities of applications at that time (lack of experimental data for a substantial number of those interesting open shell molecules of this type and little demand from the plasma simulation community), the goals of the computer codes were relatively modest. In particular, they allowed only to carry out the dynamics involving the unpaired electron (all the other 

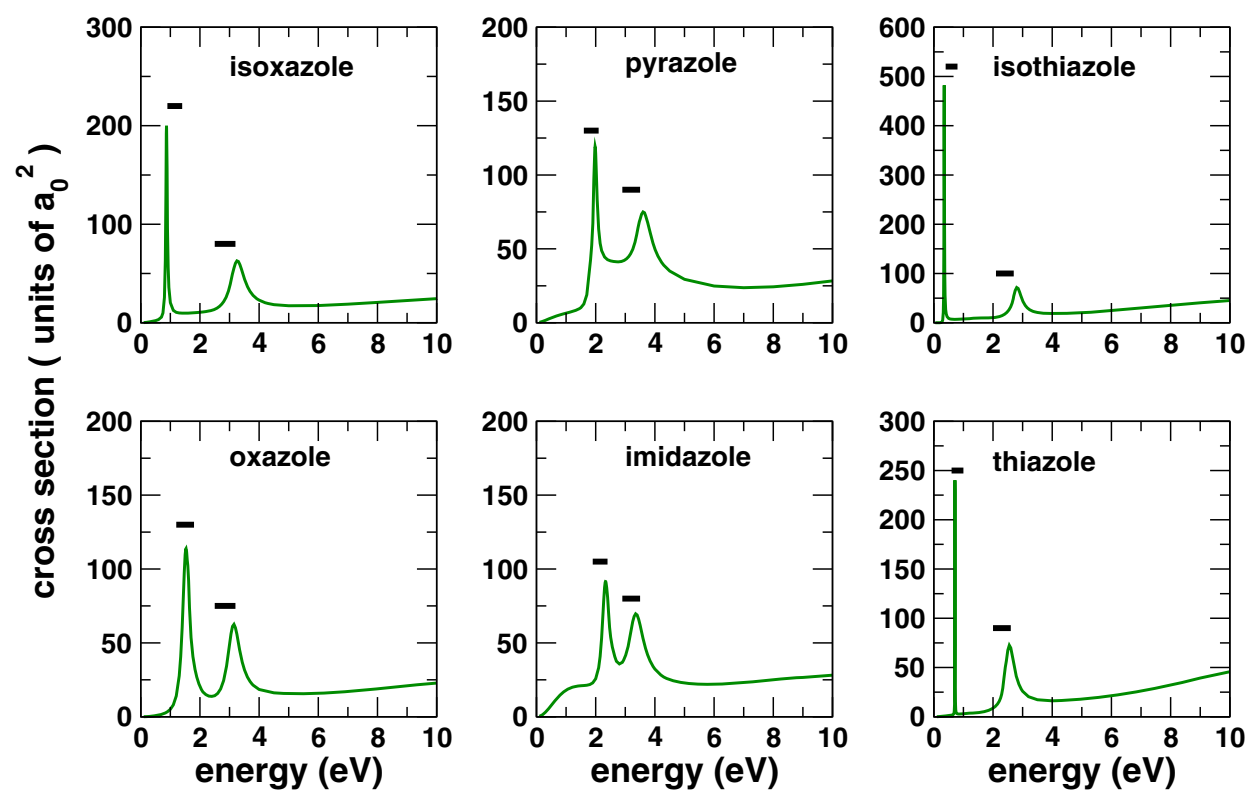

Fig. 12. Integral cross sections for the $A^{\prime \prime}$ symmetry for the aza-derivatives of furan, pyrrole and thiophene. Adapted from reference [106].

Table 4. Negative ion spectra of uracil (U), 5-fluorouracil (5$\mathrm{F}-\mathrm{U})$ and 5-chlorouracil (5-Cl-U). The energies are given in units of $\mathrm{eV}$, where bound and resonant states are indicated by negative and positive values, respectively. The theoretical results (thr) were obtained from SMCPP calculations [57] (resonances and valence bound states) and from $\operatorname{CCSD}(\mathrm{T})$ calculations for the dipole bound states (DBS [112]). The experimental data (exp) were obtained from electron transmission (resonances [114]) and photodetachment (DBS [115]) measurements. Non visible (nv) anion states are also indicated.

\begin{tabular}{ccccrrc}
\hline & & DBS & $\pi_{1}^{*}$ & \multicolumn{1}{c}{$\pi_{2}^{*}$} & \multicolumn{1}{c}{$\pi_{3}^{*}$} & $\sigma_{\mathrm{CX}}^{*}$ \\
\hline $\mathrm{U}$ & thr & -0.065 & 0.14 & 1.76 & 4.83 & \\
& $\exp$ & -0.090 & 0.22 & 1.58 & 3.83 & \\
\hline $5-\mathrm{F}-\mathrm{U}$ & thr & -0.067 & 0.09 & 1.70 & 4.97 & $\mathrm{nv}$ \\
& $\exp$ & - & 0.17 & 1.5 & 4.1 & $\mathrm{nv}$ \\
\hline \multirow{2}{*}{$5-\mathrm{Cl}-\mathrm{U}$} & thr & -0.070 & -0.23 & 1.63 & 4.59 & 2.5 \\
& $\exp$ & - & $\mathrm{nv}$ & 1.3 & 3.6 & 1.8 \\
\hline
\end{tabular}

electrons were kept frozen). As a result, the application were limited to energies where polarization effects were not too important. The first application was for NO [123], and it included spin flip cross sections. These results supported our previous explanation that molecular orientational averaging can smooth the interference pattern present in atomic cases, and produce spin-flip DCS's in accord to the experiments of reference [118]. Following this application, we employed that computer program version to calculate cross sections for a molecular radical $\mathrm{CF}_{3}$ [124], as present in $\mathrm{CF}_{4}$ plasmas.

\subsection{Electronic excitation and elastic scattering under the influence of multichannel coupling effects}

The first applications of the SMCPP in electronic excitation of molecules, by electron impact, considered small

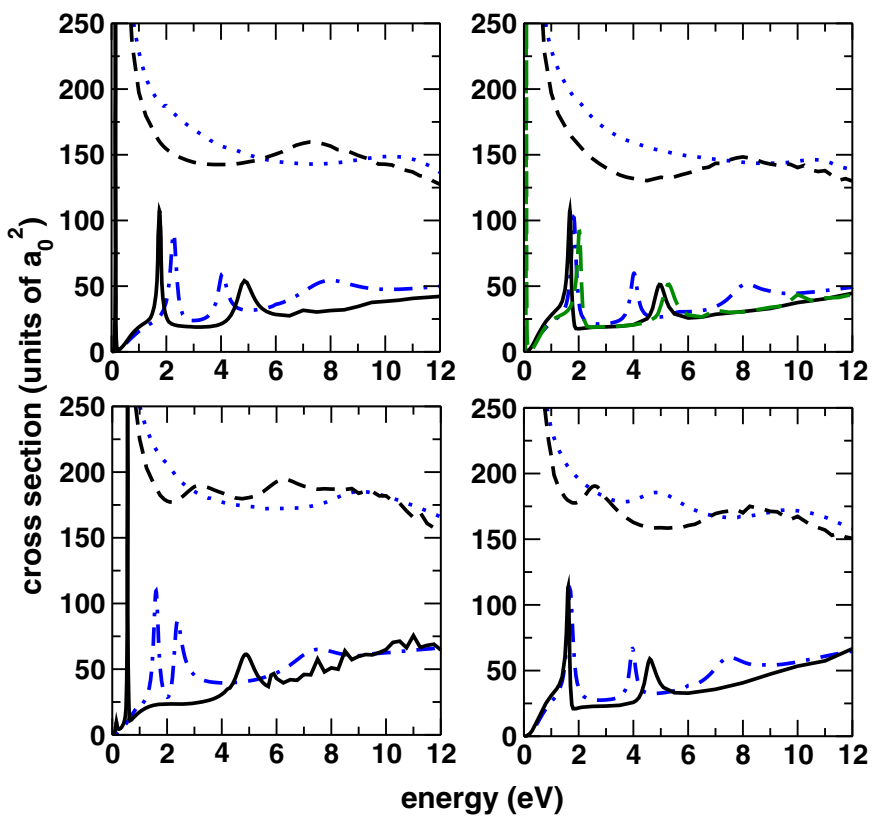

Fig. 13. Integral cross sections for uracil (top left), 5-F-uracil (top right), 5-Cl-uracil (bottom right) and 2-S-uracil (bottom left). In all panels, the back dashed lines and blue dotted lines are the SE and SEP approximations to the $A^{\prime}$ symmetry component. The solid black lines and the blue chain lines are the SE and SEP approximations to the $A^{\prime \prime}$ symmetry component. For 5-F-uracil the long-dashed green line ( $A^{\prime \prime}$ component) was obtained with a smaller CSF space.

polyatomic molecules such as formaldehyde, in a three state calculation [36], and methane, silane, germane, stannane and plumbane, in a two-state calculation [125]. These calculations, following the study for electron- $\mathrm{H}_{2}$ scattering [126], aimed to compare the all-electron results with 
the results obtained with the SMCPP method, in order to verify the efficacy of the pseudopotentials in inelastic calculations. In general, the excitation cross sections obtained considering the all-electron and pseudopotentials approaches were in very good agreement. All these applications employed IVOs to describe the excited states of the target molecules. After this successful verification, we carried out a 25-state calculation for $\mathrm{e}^{-}-\mathrm{Na}_{2}$ scattering [127], using the IVO strategy described previously. We found good agreement below $\approx 4 \mathrm{eV}$ (all 25 channels are open at $3.6 \mathrm{eV}$ ) with the experimental total cross section. Above $4 \mathrm{eV}$ our TCS was much lower in magnitude suggesting the importance of inclusion of more electronic channels.

The first attempt to investigate the influence of the multichannel coupling effect in electron-molecule collisions, through the use of the MOBSCI strategy, involved the study of the electronic excitation (from the ground state to the lowest lying triplet and singlet states) of $\mathrm{H}_{2}[38,128]$ and $\mathrm{N}_{2}[129,130]$ by electron impact. In both cases, for a particular excitation process, the open channel space was composed by the ground state, by the first triplet and singlet states of a given symmetry (more specifically, the symmetry of the excited state involved in the transition) and by the pseudo states arising from the SCI description of the target. Therefore, as a result of the use of the MOBSCI strategy, the scattering calculations in these studies were carried out at a $5 \mathrm{ch}$-sep (or at a $9 \mathrm{ch}-$ sep, for degenerate states) level of approximation. The agreement of the electronic excitation cross sections obtained according to this procedure, with the experimental results, typically ranges from good to reasonable, depending on the specific transition and system that are being considered. Following these studies, we concentrated our efforts to systems with triplets states well separated from the rest of the electronic spectra. Our expectation was that only a few states would be necessary in $P$ space to describe these electronic excitation cross sections, at low energies (around the threshold). We carried out calculations for electronic excitation of the first triplet states of furan [131] and ethylene [132], and learned that these cross sections were very sensitive to polarization effects. In both systems, shape resonances that show up above the triplet threshold in the $N_{\text {open }}$ close-coupling static-exchange approximation, would move below threshold if polarization

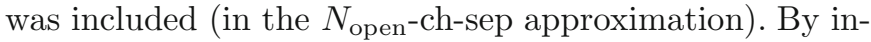
cluding polarization effects our excitation cross sections became in very good agreement with experiments, but the comparison got worse by increasing the impact energy. By increasing the energy, polarization gets less important but as we discuss below multichannel effects become more important.

Subsequent applications of the MOBSCI strategy involving small polyatomic molecules, provide a clear indication that a very large number (i.e., a much bigger number than the ones considered in the previously mentioned studies) of electronic states should be included in the expansion of the open channel space in order to achieve (or, at least, move towards obtaining) converged elastic and electronic excitation cross sections. In what follows we in-

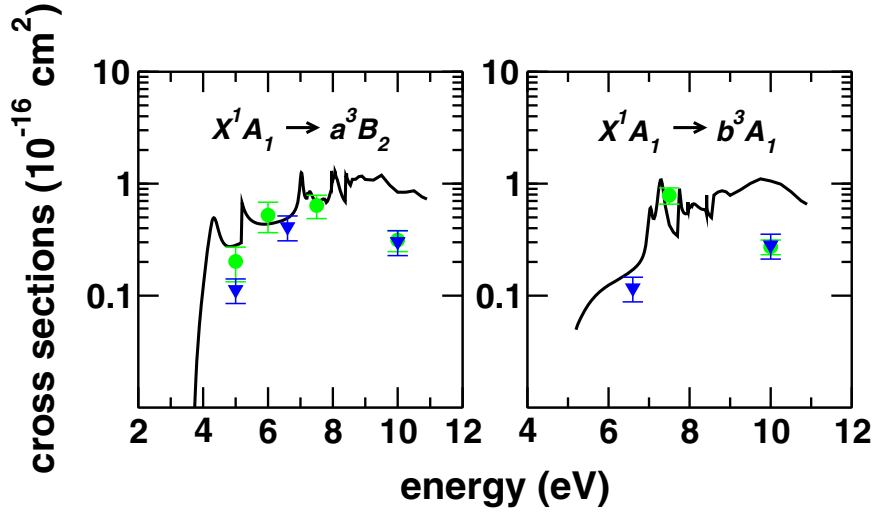

Fig. 14. Integral cross sections for the $\mathrm{X}^{1} \mathrm{~A}_{1} \rightarrow \tilde{a}^{3} \mathrm{~B}_{2}$ (left panel) and $\mathrm{X}^{1} \mathrm{~A}_{1} \rightarrow \tilde{b}^{3} \mathrm{~A}_{1}$ (right panel) electronic excitation of furan $\left(\mathrm{C}_{4} \mathrm{H}_{4} \mathrm{O}\right)$ by electron impact. Solid line, theoretical results from reference [133] obtained up to the 9ch-sep level of approximation; circles, experimental data from reference [133]; triangles, experimental data from reference [135]. Adapted from reference [133].

tend to illustrate this finding by presenting three sets of results, each one of them obtained in calculations in which the multichannel coupling effect was treated to a growing level of complexity.

In Figure 14 we present integral cross sections for the $\mathrm{X}^{1} \mathrm{~A}_{1} \rightarrow \tilde{a}^{3} \mathrm{~B}_{2}$ and $\mathrm{X}^{1} \mathrm{~A}_{1} \rightarrow \tilde{b}^{3} \mathrm{~A}_{1}$ electronic transitions in furan induced by electron impact [133]. In that case, the scattering calculations were performed at a 9ch-sep level of approximation. As can be seen, the energy dependence of the ICS is strongly affected by the presence of several spurious peaks, whose origin is associated to states that become energetically accessible but were treated as closed channels in the calculations. In spite of this, the agreement with the experimental data is reasonable, in the sense that the calculated ICS reproduces the gradual rise in the region comprised between near-threshold to around $8 \mathrm{eV}$. For higher energies, the agreement is definitely poorer. The corresponding differential cross sections obviously follow the same trends discussed above, and representative DCSs at the energy of $7.5 \mathrm{eV}$ are shown in Figure 15 for each one of the excitation processes considered in that work. Much more encouraging results were obtained when the complementary investigation for the elastic scattering of electrons by furan under the influence of the multichannel coupling effect was considered, as shown Figure 16. At $1 \mathrm{eV}$ only the elastic channel is open under a fixed nuclei framework and the DCS obtained at the 1ch-sep level of approximation is in good agreement with the experiments, while at $5 \mathrm{eV}$ the results obtained at the 1 ch-sep and the 2ch-sep levels of approximation are almost identical and in excellent agreement with the experimental data. As the impact energy is increased, the importance of the multichannel coupling effect is clearly highlighted, as expected. In fact, for the energies of 20 and $50 \mathrm{eV}$ the DCSs obtained at the 9ch-sep level of approximation are in much better agreement with experiments than the those obtained at the 1ch-sep level of approximation. Finally, it 


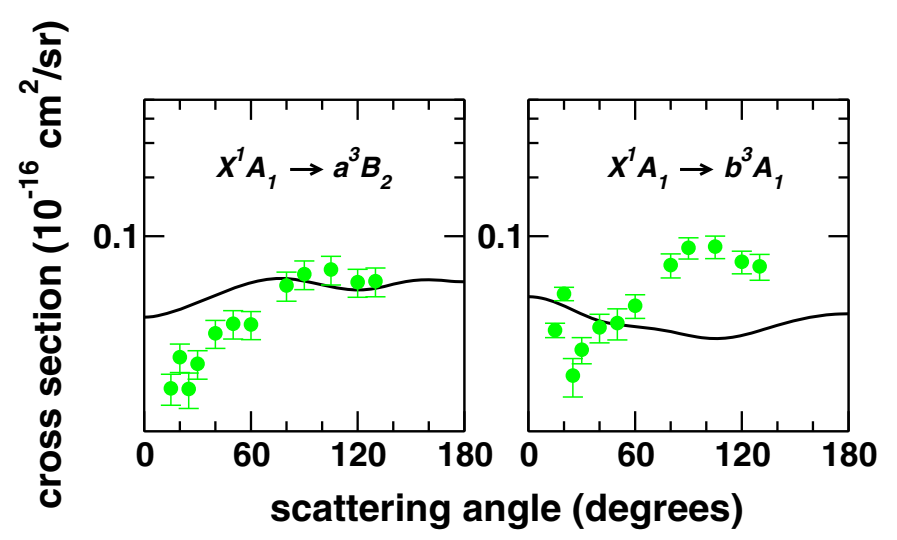

Fig. 15. Differential cross sections for the $\mathrm{X}^{1} \mathrm{~A}_{1} \rightarrow \tilde{a}^{3} \mathrm{~B}_{2}$ (left panel) and $\mathrm{X}^{1} \mathrm{~A}_{1} \rightarrow \tilde{b}^{3} \mathrm{~A}_{1}$ (right panel) electronic excitation of furan $\left(\mathrm{C}_{4} \mathrm{H}_{4} \mathrm{O}\right)$ by electron impact at $7.5 \mathrm{eV}$. Solid line, theoretical results from reference [133] obtained up to the 9ch-sep level of approximation; circles, experimental data from reference [133]. Adapted from reference [133].

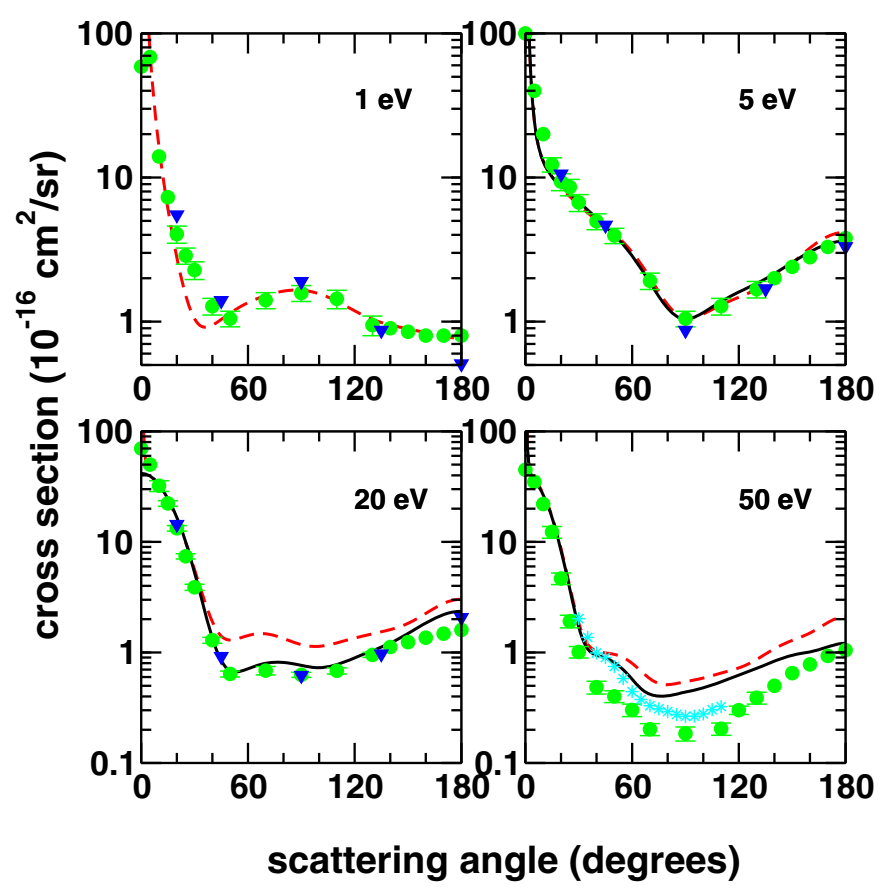

Fig. 16. Differential cross sections for elastic electron scattering from furan $\left(\mathrm{C}_{4} \mathrm{H}_{4} \mathrm{O}\right)$ at the energies of 1, 5, 20 and $50 \mathrm{eV}$. Solid line, theoretical results from reference [133] obtained up to the 9ch-sep level of approximation; dashed line, theoretical results from reference [134] obtained at the 1ch-sep level of approximation; circles, experimental data from reference [134]; triangles, experimental data from reference [135]; stars, experimental data from reference [136]. Adapted from references $[133,134]$.

is also important to mention that the difference between the 9ch-sep result and the experimental results, at $50 \mathrm{eV}$, points out that the convergence of the cross sections due to multichannel coupling has not been reached within the 9ch-sep scheme for this energy.

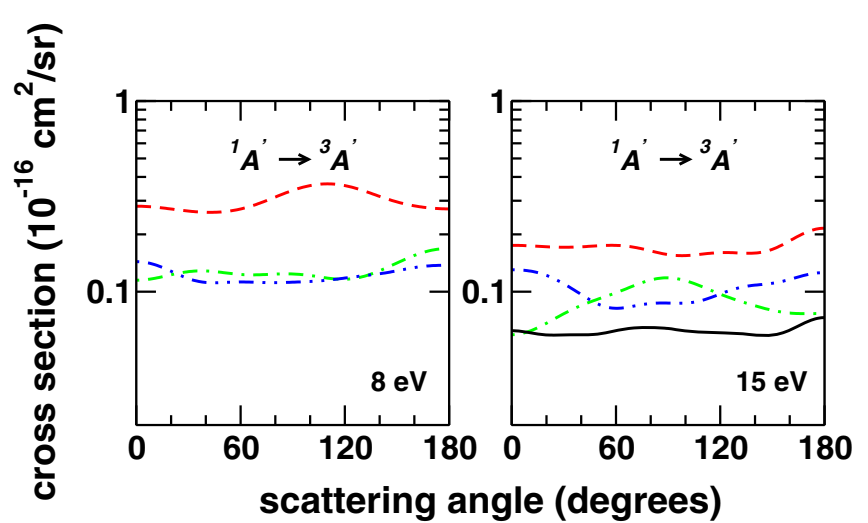

Fig. 17. Differential cross sections for the ${ }^{1} \mathrm{~A}^{\prime} \rightarrow{ }^{3} \mathrm{~A}^{\prime}$ electronic excitation of phenol $\left(\mathrm{C}_{6} \mathrm{H}_{5} \mathrm{OH}\right)$ by electron impact at the energies of $8 \mathrm{eV}$ (left panel) and $15 \mathrm{eV}$ (right panel). Dashed (red) line, dash-dotted (green) line, dash-dot-dotted (blue) line and solid (black) line, theoretical results from reference [137] obtained at the $3 \mathrm{ch}$-sep, $8 \mathrm{ch}$-sep, $13 \mathrm{ch}$-sep and $23 \mathrm{ch}$-sep levels of approximation, respectively. Adapted from reference [137].

More recently, the development of the parallel version for the SMCPP computational codes allowed us to explore the effects of multichannel coupling in an even more comprehensive way. Latest applications involving phenol [137] and ethylene [138] molecules were carried out with the inclusion of 33 and 45 electronically open channels, respectively. With regard to the use of the MOBSCI strategy, these are the most sophisticated calculations performed to date. In Figure 17 we present the cross sections for the electronic excitation from ground state to the first triplet state $\left({ }^{3} \mathrm{~A}^{\prime}\right)$ of phenol by electron impact. Comparison between results obtained at the 3ch-sep, 8ch-sep, 13ch-sep and 23ch-sep levels of approximation indicate that the probability flux to the ${ }^{3} \mathrm{~A}^{\prime}$ state decreases as more channels are included in the calculations and, although not shown here, the same trend was observed for higher energies. Figure 18 displays the DCSs for elastic electron scattering from phenol at the energies of 5, 10, 20 and $40 \mathrm{eV}$. These results indicate that the difference between cross sections obtained at the $1 \mathrm{ch}$-sep and the $N_{\text {open }}$ ch-sep levels of approximation (with $N_{\text {open }}=3$ for $5 \mathrm{eV}, 21$ for $10 \mathrm{eV}, 23$ for $20 \mathrm{eV}$ and 33 for $40 \mathrm{eV}$ ) becomes larger as the impact energy increases. In addition, at higher energies, the agreement with the experimental data for benzene [141] is improved as more channels are included in the calculations. Basically, the same remarks apply to the results obtained for the elastic and electronically inelastic electron collisions with ethylene. In short, the elastic DCSs presented in Figure 19 denote that the results obtained within the $45 \mathrm{ch}$-sep scheme are systematically in much better agreement with the experimental data if compared to those obtained at the 1ch-sep level of approximation. The same is true for the $\mathrm{X}^{1} \mathrm{~A}_{g} \rightarrow \tilde{a}^{3} \mathrm{~B}_{1 u}$ electronic excitation DCSs, except that now the agreement with experiments is good only for energies near to the threshold of the $\tilde{a}^{3} \mathrm{~B}_{1 u}$ excited state. For higher energies, the calculated DCSs reproduce in qualitative terms the shape 


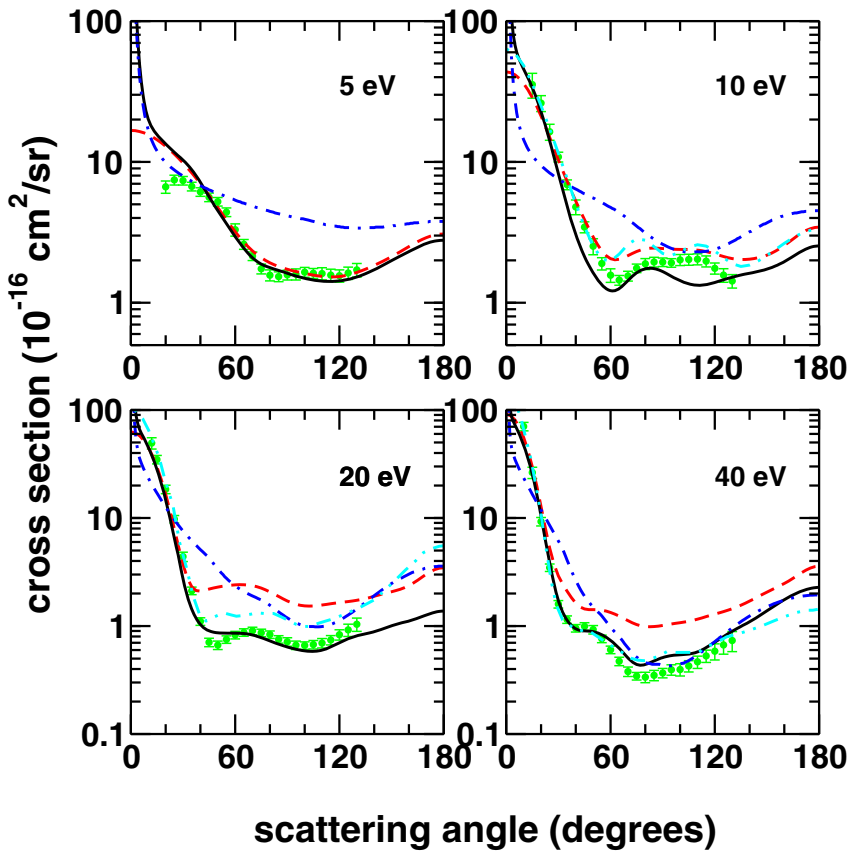

Fig. 18. Differential cross sections for elastic electron scattering from phenol $\left(\mathrm{C}_{6} \mathrm{H}_{5} \mathrm{OH}\right)$ at the energies of $5,10,20$ and $40 \mathrm{eV}$. Solid (black) line, theoretical results from reference [137] obtained up to a 33ch-sep level of approximation; dashed (red) line, theoretical results from reference [137] obtained at the 1ch-sep level of approximation; dash-dotted (blue) line, IAM-SCAR theoretical result from reference [137]; dash-dot-dotted (cyan) line SVIM theoretical result for benzene from reference [142]; circles, experimental data from reference [141]. Adapted from reference [137].

but are larger in magnitude than the experimental cross sections by a factor of around 3 .

Finally, it is important to mention that these results highlighted the fact that for any system having a high density of energetically accessible states, as is the case for the molecules considered here, the rate of convergence of the multichannel coupling for electronically inelastic processes should be very slow. In order to overcome this difficulty, and aiming at future applications using a redesigned computer code, we tested the possibility of defining a fictitious arbitrary threshold at $10 \mathrm{eV}$, meaning that all states opening up at energies between 10 and $18 \mathrm{eV}$ were treated as degenerate open states with a threshold at $10 \mathrm{eV}$. Upon doing so, for the remaining 27 states ( 45 minus the ground state and the 17 excited states below $10 \mathrm{eV}$ ) we could use the same integrals (Coulomb potential integrals involving Cartesian Gaussians and plane waves) to evaluate the numerators and the Green function appearing in equation (2). Through the use of the same on-shell energy for all these states, the number of integrals is substantially decreased. The results for this approximation, represented by the solid (brown) line in Figures 19 and 20, are quite satisfactory if compared to those obtained at the 45ch-sep level of approximation. The use of the fictitious thresholds, therefore, represents a very promising strategy that will be further explored in future applications of the SMCPP method for studying electron-molecule collisions.

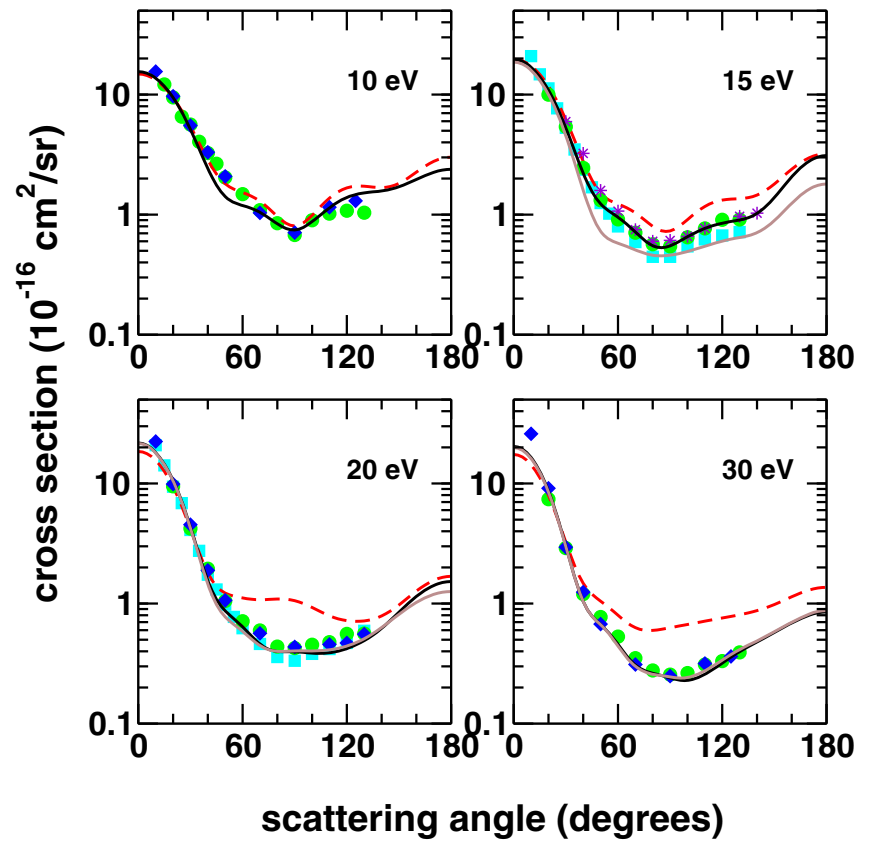

Fig. 19. Differential cross sections for elastic electron scattering from ethylene $\left(\mathrm{C}_{2} \mathrm{H}_{4}\right)$ at the energies of 10, 15, 20 and $30 \mathrm{eV}$. Solid (black) line, theoretical results from reference [138] obtained up to a $45 \mathrm{ch}$-sep level of approximation; dashed (red) line, theoretical results from reference [138] obtained at the 1ch-sep level of approximation; solid (brown) line, theoretical results obtained up to a $45 \mathrm{ch}$-sep with fictitious thresholds level of approximation from reference [138]; circles, diamonds, squares and stars, experimental data from references [143-146], respectively.

The existing discrepancies observed in these phenol and ethylene studies help to illustrate the difficulties in readily establishing reliable cross sections of polyatomic molecules by low-energy electrons. In addition they highlight the very special role played by the multichannel coupling effect, in particular, showing how the number of excited states included in the open channel space impacts upon the convergence of the cross sections at intermediate and higher collision energies.

An alternative to the use of ab initio description of the multichannel effect in the elastic channel is the use of model complex (absorption) potential, as that developed by Staszewska et al. [147-149]. This potential mimics the flux loss from the elastic channel to all other channels (excitation, ionization, etc.), and as a result the elastic cross sections agree well with the experiment even at higher energies. The potential of Staszewska et al. [147-149], and its improvements [150-152], have been successfully employed in cross section calculations for collisions of electrons by molecules $[18,19,153]$.

\section{Conclusions}

We have presented recent advances in the application of the Schwinger multichannel method to collisions of lowenergy electrons with molecular targets. In the last years, 


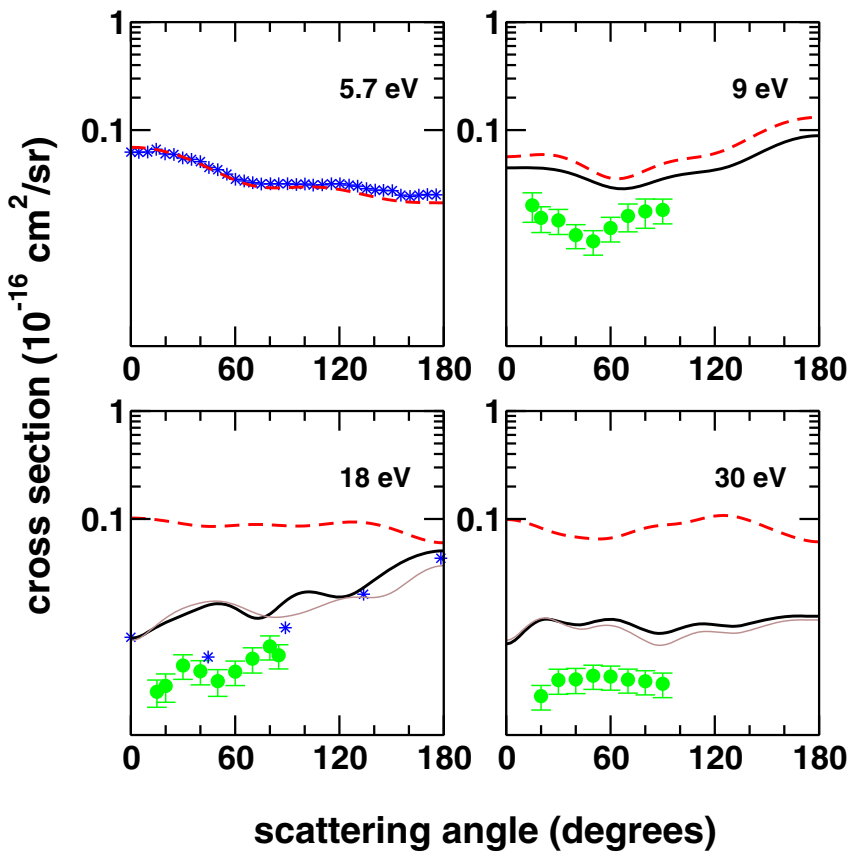

Fig. 20. Differential cross sections for the $\mathrm{X}^{1} \mathrm{~A}_{g} \rightarrow \tilde{a}^{3} \mathrm{~B}_{1 u}$ electronic excitation of ethylene $\left(\mathrm{C}_{2} \mathrm{H}_{4}\right)$ by electron impact at the energies of 5.7, 9, 18 and $30 \mathrm{eV}$. Solid (black) line, theoretical results from reference [138] obtained up to a 45ch-sep level of approximation; dashed (red) line, theoretical results from reference [138] obtained at the 2ch-sep level of approximation; circles, experimental data from reference [139]; stars, experimental data from reference [140]. Adapted from reference [138].

substantial progress related to our ability in determining reliable cross sections has been achieved through the development of efficient computational strategies especially designed with the aim of dealing with some of the current challenges posed to the community.

An important step towards this direction was the consolidation of the use of pseudopotentials in the study of electron-molecule collisions, since this achievement enabled us to handle heavy targets (molecular targets containing any atom of the periodic table) by performing calculations with a reduced computational cost compared to the all-electron calculations. This methodology has been employed to compute cross sections for collisions of slow electrons by a large number of molecular targets, in very good agreement with results from different all-electron methods and with the experiments. It has also been employed to investigate the shape resonance spectra of large molecules.

In recent investigations concerning electron collisions with furan and ethylene molecules we found that a proper treatment of polarization effects was essential for an accurate description of the electronic excitation, improving the agreement between the calculated and measured cross sections. That improvement is related to the fact that the electronic excitation cross sections are dominated by the tail contribution of the shape resonances present in the elastic channel. In summary, these theoretical studies suggest that for molecular systems supporting res- onances near low-energy electronic thresholds, standard close-coupling calculations may produce inaccurate results in view of the improper treatment of the polarization effects, which will give rise to misplaced resonances in the elastic channel.

Another important finding raised by studies involving furan, ethylene and, more recently, phenol molecules is related to the analysis of the influence of multichannel coupling effects upon calculated elastic and electronic excitation cross sections. Comparison among theoretical results obtained with different channel-coupling schemes, clearly indicates that convergence of the elastic cross sections, in terms of the number of excited states included in the open-channel space, is relatively quickly achieved for energies below $30 \mathrm{eV}$. For the electronic excitation processes, our calculations revealed that at energies near to the thresholds of the excited states we are in good agreement with the experiment. However, for higher energies our results lie systematically above the experimental data, indicating that probably more open channels would be needed in order to lower the magnitude of the computed inelastic DCSs. This would be a problem, considering that molecular systems typically present a high density of energetically accessible states. An interesting result for phenol [137] was that for a fixed $(N+1)$-particle configuration space, the TCS for the 1ch-sep came very close to the $33 \mathrm{ch}-$ sep calculation. This indicates that as we open more and more channels through the $P$ projector, a strong flux distribution takes place among the channels. Convergence with respect to open channels is an important challenge. Aiming to address this question in a more computationally efficient way, we also introduced a simple but rather effective method of representing multichannel-coupling effects through the use of fictitious thresholds. Exploratory studies have shown that it provides DCSs in agreement with the case where all thresholds are placed in the correct positions. That is, through the use of these fictitious thresholds (which allow the creation of groups of degenerate states), a scattering calculation can be carried out with the same energy dependent two-electron integrals for each group. All channels lying within a pre-determined energy interval are treated as a single group of degenerate channels, capable of reproducing the results of a calculation in which the number of coupled channels would demand a much larger set of integrals. We thus intend to make a much more intense use of the fictitious thresholds strategy in the future.

The authors acknowledge support from Brazilian Agency Conselho Nacional de Desenvolvimento Científico e Tecnológico (CNPq). R.F. da C., M.T. do N.V. and M.A.P.L. also acknowledge support from Fundação de Amparo à Pesquisa do Estado de São Paulo (FAPESP). M.H.F.B. also acknowledges support from FINEP (under project CT-Infra) and computational support from Professor Carlos M. de Carvalho at LFTC-DFis-UFPR and at LCPAD-UFPR. The authors acknowledge computational support from CENAPAD-SP. We would like to express our gratitude to Prof. Luiz G. Ferreira for his invaluable collaboration over the years and his fundamental 
contribution in the SMCPP implementation, in the formulation of a method for basis set generation and collaboration in several papers. We would also like to thank Prof. Fernando J. da Paixão, Dr. Alexandra P.P. Natalense, Dr. Cláudio S. Sartori, Dr. Roberto B. Diniz, Prof. David L. Azevedo, Prof. Antônio S. Oliveira, Dr. Adriana R. Lopes, Dr. Eliane M. de Oliveira, Prof. Thiago C. de Freitas, Prof. Sergio d'A. Sanchez, Dr. Andreia N.S. Hisi, Prof. Emerson Joucoski, and the graduate students Fábris Kossoski, Josué S. dos Santos, Alessandra de S. Barbosa, Diego F. Pastega, Flávio M. da Silva, for their important contribution to several papers.

\section{References}

1. P.V. Johnson, C.P. Malone, M.A. Khakoo, J.W. McConkey, I. Kanik, J. Phys.: Conf. Ser. 88, 012069 (2007)

2. L. Campbell, M. Brunger, Plasma Sources Sci. Technol. 22, $013002(2013)$

3. W.M. Huo, V. McKoy, M.A.P. Lima, T.L. Gibson, Thermophysical Aspects of Reentry Flow, edited by J. Moss, C. Scott (AIAA, New York, 1986), Vol. 103, pp. $152-196$

4. M.A. Lieberman, A.J. Lichtenberg, Principles of Plasma Discharges and Materials Processing (John Wiley \& Sons, 1994)

5. W.N.G. Hitchon, Plasma Processes for Semiconductor Fabrication (Cambridge University Press, 1999)

6. L.G. Christophorou, J.K. Olthoff, J. Phys. Chem. Ref. Data 28, 967 (1999)

7. J.-S. Yoon, M.-Y. Song, H. Kato, M. Hoshino, H. Tanaka, M.J. Brunger, S.J. Buckman, H. Cho, J. Phys. Chem. Ref. Data. 39, 033106 (2010)

8. L. Sanche, Eur. Phys. J. D 35, 367 (2005)

9. W.F. van Dorp, Phys. Chem. Chem. Phys. 14, 16753 (2012)

10. W.F. van Dorp, X. Zhang, B.L. Feringa, T.W. Hansen, J.B. Wagner, J.Th.M. de Hosson, ACS Nano 6, 10076 (2012)

11. A.J. Ragauskas et al., Science 311, 484 (2006)

12. J. Amorim, C. Oliveira, J.A. Souza-Corrêa, M.A. Ridenti, Plasma Processes Polym. 10, 670 (2013)

13. E.M. de Oliveira, R.F. da Costa, S. d'A. Sanchez, A.P.P. Natalense, M.H.F. Bettega, M.A.P. Lima, M.T. do N. Varella, Phys. Chem. Chem. Phys. 15, 1682 (2013)

14. M.T. do N. Varella, Rev. Phys. 1, 45 (2000)

15. N.F. Lane, Rev. Mod. Phys. 52, 29 (1980)

16. F.A. Gianturco, D.G. Thompson, J. Phys. B 9, 1383 (1976)

17. G.L.C. de Souza, A.S. dos Santos, R.R. Lucchese, L.E. Machado, L.M. Brescansin, H.V. Manini, I. Iga, M.-T. Lee, Chem. Phys. 393, 19 (2012)

18. P. Palihawadana, J. Sullivan, M. Brunger, C. Winstead, V. McKoy, G. García, F. Blanco, S. Buckman, Phys. Rev. A 84, 062702 (2011)

19. M.C. Fuss, A.G. Sanz, F. Blanco, J.C. Oller, P. LimãoVieira, M.J. Brunger, G. García, Phys. Rev. A 88, 042702 (2013)

20. A.W. Fliflet, V. McKoy, Phys. Rev. A 21, 1863 (1980)

21. S. Chung, C.C. Lin, Phys. Rev. A 17, 1874 (1978)

22. L.A. Collins, B.I. Schneider, Phys. Rev. A 27, 101 (1983)

23. P.G. Burke, C.J. Noble, Comment. At. Mol. Phys. 12 , $301(1983)$
24. K. Takatsuka, V. McKoy, Phys. Rev. A 24, 2473 (1981)

25. K. Takatsuka, V. McKoy, Phys. Rev. A 30, 1734 (1984)

26. B. Schneider, T.N. Rescigno, Phys. Rev. A 37, 3749 (1988)

27. N.S. Ostund, Chem. Phys. Lett. 34, 419 (1975)

28. D.A. Levin, A.W. Fliflet, M. Ma, V. McKoy, J. Comput. Phys. 28, 416 (1978)

29. D.K. Watson, R.R. Lucchese, V. McKoy, T.N. Rescigno, Phys. Rev. A 21, 738 (1980)

30. M.A.P. Lima, T.L. Gibson, K. Takatsuka, V. McKoy, Phys. Rev. A 30, 1741 (1984)

31. T.L. Gibson, M.A.P. Lima, K. Takatsuka, V. McKoy, Phys. Rev. A 30, 3005 (1984)

32. M.A.P. Lima, T.L. Gibson, W.M. Huo, V. McKoy, Phys. Rev. A 32, 2696 (1985)

33. W.M. Huo, T.L. Gibson, M.A.P. Lima, V. McKoy, Phys. Rev. A 36, 1632 (1987)

34. W.M. Huo, M.A.P. Lima, T.L. Gibson, V. McKoy, Phys. Rev. A 36, 1642 (1987)

35. C.L. Winstead, P.F. Hipes, M.A.P. Lima, V. McKoy, J. Chem. Phys. 94, 5455 (1991)

36. M.H.F. Bettega, L.G. Ferreira, M.A.P. Lima, Phys. Rev. A 47, 1111 (1993)

37. M.A.P. Lima, L.M. Brescansin, A.J.R. da Silva, C.L. Winstead, V. McKoy, Phys. Rev. A 41, 327 (1990)

38. R.F. da Costa, F.J. da Paixão, M.A.P. Lima, J. Phys. B 37, L129 (2004)

39. J.S. dos Santos, R.F. da Costa, M.T. do N. Varella, J. Chem. Phys. 136, 084307 (2012)

40. M.A.P. Lima, V. McKoy, Phys. Rev. A 38, 501 (1988)

41. H. Feshbach, Ann. Phys. 5, 357 (1958)

42. H. Feshbach, Ann. Phys. 19, 287 (1962)

43. B.I. Schneider, L.A. Collins, J. Phys. B 18, L857 (1985)

44. J.S.E. Germano, M.A.P. Lima, Phys. Rev. A 47, 3976 (1993)

45. C. Winstead, V. McKoy, Adv. At. Mol. Opt. Phys. 36, 183 (1996)

46. C. Winstead, V. McKoy, Comput. Phys. Commun. 128, $386(2000)$

47. C.J. Joachain, Quantum Collisions Theory (NorthHolland, 1975)

48. W.J. Hunt, W.A. Goddard III, Chem. Phys. Lett. 3, 414 (1969)

49. G. Bachelet, D.R. Hamann, M. Schlüter, Phys. Rev. B 46, 4199 (1982)

50. D.R. Hamann, M. Schlüter, C. Chiang, Phys. Rev. Lett. 43, 1494 (1979)

51. M.H.F. Bettega, A.P.P. Natalense, M.A.P. Lima, L.G. Ferreira, Int. J. Quantum. Chem. 60, 821 (1996)

52. M.E. Rose, Elementary Theory of Angular Momentum (John Wiley and Sons, New York 1957)

53. T.N. Rescigno, B.I. Schneider, Phys. Rev. A 45, 2894 (1992)

54. E.M. de Oliveira, R.F. da Costa, S. d'A. Sanchez, A.P.P. Natalense, M.H.F. Bettega, M.A.P. Lima, M.T. do N. Varella, Phys. Chem. Chem. Phys. 15, 1682 (2013)

55. M.T. do N. Varella, M.H.F. Bettega, A.J.R. da Silva, M.A.P. Lima, J. Chem. Phys. 110, 2452 (1999)

56. M.T. do N. Varella, M.H.F. Bettega, M.A.P. Lima, L.G. Ferreira, J. Chem. Phys. 111, 6396 (1999)

57. F. Kossoski, M.H.F. Bettega, M.T. do N. Varella, J. Chem. Phys. 140, 024317 (2014)

58. D.M. Chase, Phys. Rev. 104, 838 (1956) 
59. A.P.P. Natalense, M.H.F. Bettega, L.G. Ferreira, M.A.P. Lima, Phys. Rev. A 52, R1 (1995)

60. M.T. do N. Varella, A.P.P. Natalense, M.H.F. Bettega, M.A.P. Lima, Phys. Rev. A 60, 3684 (1999)

61. M.H.F. Bettega, A.P.P. Natalense, M.A.P. Lima, L.G. Ferreira, J. Phys. B 36, 1263 (2003)

62. K. Sunohara, M. Kitajima, H. Tanaka, M. Kimura, H. Cho, J. Phys. B 36, 1843 (2003)

63. L.R. Hargreaves, J.R. Brunton, T.M. Maddern, M.J. Brunger, J. Chem. Phys. 142, 124310 (2015)

64. M. Kitajima, M. Okamoto, K. Sunohara, H. Tanaka, H. Cho, S. Samukawa, S. Eden, N.J. Mason, J. Phys. B 35, 3257 (2002)

65. H. Cho, M.Y. Song, J.S. Yoon, M. Hoshino, H. Tanaka, J. Phys. B 43, 135205 (2010)

66. J.R. Francis-Staite, B.A. Schmerl, M.J. Brunger, H. Kato, S.J. Buckman, Phys. Rev. A 81, 022704 (2010)

67. M.H.F. Bettega, Phys. Rev. A 84, 052725 (2011)

68. H.-X. Wan, J.H. Moore, J.A. Tossel, J. Chem. Phys. 91, $7340(1989)$

69. P. Możejko, G. Kasperski, Cz. Szmytkowski, A. Zecca, G.P. Karwasz, L. Del Longo, R.S. Brusa, Eur. Phys. J. D 6, $481(1999)$

70. Y. Nakano, M. Hoshino, M. Kitajima, H. Tanaka, M. Kimura, Phys. Rev. A 66, 032714 (2002)

71. C. Makochekanwa, H. Kawate, O. Sueoka, M. Kimura, M. Kitajima, M. Hoshino, H. Tanaka, Chem. Phys. Lett. 368, $82(2003)$

72. Cz. Szmytkowski, S. Kwitnewski, J. Phys. B 35, 3781 (2002)

73. Cz. Szmytkowski, S. Kwitnewski, J. Phys. B 36, 2129 (2003)

74. A.R. Lopes, M.H.F. Bettega, Phys. Rev. A 67, 032711 (2003)

75. S. d'A. Sanchez, A.R. Lopes, M.H.F. Bettega, M.A.P. Lima, L.G. Ferreira, Phys. Rev. A 71, 062702 (2005)

76. A.R. Lopes, M.A.P. Lima, L.G. Ferreira, M.H.F. Bettega, Phys. Rev. A 69, 014702 (2004)

77. A.R. Lopes, M.H.F. Bettega, M.A.P. Lima, L.G. Ferreira, J. Phys. B 37, 997 (2004)

78. A.R. Lopes, M.H.F. Bettega, M.T. do N. Varella, M.A.P. Lima, Eur. Phys. J. D 37, 385 (2006)

79. C. Makochekanwa, H. Kato, M. Hoshino, H. Tanaka, H. Kubo, M.H.F. Bettega, A.R. Lopes, M.A.P. Lima, L.G. Ferreira, J. Chem. Phys. 124, 024323 (2006)

80. L.G. Ferreira, A.R. Lopes, M.A.P. Lima, M.H.F. Bettega, J. Phys. B 39, 1045 (2006)

81. M.A. Khakoo, J. Muse, H. Silva, M.C.A. Lopes, C. Winstead, V. McKoy, E.M. de Oliveira, R.F. da Costa, M.T. do N. Varella, M.H.F. Bettega, M.A.P. Lima, Phys. Rev. A 78, 062714 (2008)

82. M.H.F. Bettega, C. Winstead, V. McKoy, Phys. Rev. A 82, 062709 (2010)

83. M.H.F. Bettega, C. Winstead, V. McKoy, A. Jo, A. Gauf, J. Tanner, L.R. Hargreaves, M.A. Khakoo, Phys. Rev. A 84, 042702 (2011)

84. K. Fedus, C. Navarro, L.R. Hargreaves, M.A. Khakoo, F.M. Silva, M.H.F. Bettega, C. Winstead, V. McKoy, Phys. Rev. A 90, 032708 (2014)

85. A. Pelc, W. Sailer, P. Scheier, M. Probst, N.J. Mason, E. Illenberger, T.D. Märk, Chem. Phys. Lett. 361, 277 (2002)
86. A. Pelc, W. Sailer, P. Scheier, N.J. Mason, T.D. Märk, Eur. Phys. J. D 20, 441 (2002)

87. V. Vizcaino, M. Jelisavcic, J.P. Sullivan, S.J. Buckman, New J. Phys. 8, 85 (2006)

88. F.A. Gianturco, R.R. Lucchese, New J. Phys. 6, 66 (2004)

89. F.A. Gianturco, R.R. Lucchese, Eur. Phys. J. D 39, 399 (2006)

90. T.N. Rescigno, C.S. Trevisan, A.E. Orel, Phys. Rev. Lett. 96, 213201 (2006)

91. M. Allan, J. Phys. B 39, 2939 (2006)

92. M.H.F. Bettega, Phys. Rev. A 74, 054701 (2006)

93. T.C. Freitas, S. d'A. Sanchez, M.T. do N. Varella, M.H.F. Bettega, Phys. Rev. A 84, 062714 (2011)

94. M. Seydou, A. Modelli, B. Lucas, K. Konate, C. Desfrançois, J.P. Schermann, Eur. Phys. J. D 35, 199 (2005)

95. P. Cloutier, C. Sicard-Roselli, E. Escher, L. Sanche, J. Phys. Chem. B 111, 1620 (2007)

96. T.P.M. Goumans, F.A. Gianturco, F. Sebastianelli, I. Baccarelli, J.L. Rivail, J. Chem. Theory Comput. 5, 217 (2009)

97. F.F. da Silva, S. Denifl, T.D. Märk, N.L. Doltsinis, A.M. Ellis, P. Scheier, J. Phys. Chem. A 114, 1633 (2010)

98. M.H.F. Bettega, Phys. Rev. A 81, 062717 (2010)

99. T.C. Freitas, M.A.P. Lima, S. Canuto, M.H.F. Bettega, Phys. Rev. A 80, 062710 (2009)

100. T.C. Freitas, K. Coutinho, M.T. do N. Varella, M.A.P. Lima, S. Canuto, M.H.F. Bettega, J. Chem. Phys. 138, 174307 (2013)

101. E.M. Oliveira, T.C. Freitas, K. Coutinho, M.T. do N. Varella, S. Canuto, M.A.P. Lima, M.H.F. Bettega, J. Chem. Phys. 141, 051105 (2014)

102. M.H.F. Bettega, M.A.P. Lima, J. Chem. Phys. 126, 194317 (2007)

103. M.A. Khakoo, J. Muse, K. Ralphs, R.F. da Costa, M.H.F. Bettega, M.A.P. Lima, Phys. Rev. A 81, 062716 (2010)

104. E.M. de Oliveira, M.A.P. Lima, M.H.F. Bettega, S. d'A. Sanchez, R.F. da Costa, M.T. do N. Varella, J. Chem. Phys. 132, 204301 (2010)

105. R.F. da Costa, M.T. do N. Varella, M.A.P. Lima, M.H.F. Bettega, J. Chem. Phys. 138, 194306 (2013)

106. F. Kossoski, M.H.F. Bettega, J. Chem. Phys. 138, 234311 (2013)

107. A. Modelli, P.D. Burrow, J. Phys. Chem. A 108, 5721 (2004)

108. S. Gohlke, E. Illenberger, Europhys. News 33, 207 (2002)

109. S. Zamenhof, R. Degiovanni, S. Greer, Nature 181, 827 (1958)

110. P. Wardman, Clin. Oncol. 19, 397 (2007)

111. A.S. Barbosa, M.H.F. Bettega, J. Chem. Phys. 139, $214301(2013)$

112. F. Kossoski, M.T. do N. Varella, private communication

113. P. Skurski, M. Gutowski, J. Simons, Int. J. Quantum. Chem. 80, 1024 (2000)

114. A. Scheer, K. Aflatooni, G. Gallup, P. Burrow, Phys. Rev. Lett. 92, 068102 (2004)

115. J. Schiedt, R. Weinkauf, D.M. Neumark, E.W. Schlag, Chem. Phys. 239, 511 (1998)

116. J. Kopyra, H. Abdoul-Carime, F. Kossoski, M.T. do N. Varella, Phys. Chem. Chem. Phys. 16, 25054 (2014)

117. J.S. dos Santos, F. Kossoski, M.T. do N. Varella, Phys. Rev. A 90, 052713 (2014) 
118. T. Hegeman, M. Oberste-Vorth, G.F. Hanne, Phys. Rev. Lett. 66, 2968 (1991)

119. F.J. da Paixão Filho, M.A.P. Lima, V. McKoy, Phys. Rev. Lett. 68, 1698 (1992)

120. C.S. Sartori, F.J. da Paixão Filho, M.A.P. Lima, Phys. Rev. A 55, 3243 (1997)

121. C.S. Sartori, F.J. da Paixão Filho, M.A.P. Lima, Phys. Rev. A 58, 2857 (1998)

122. J. Amorim, J.L. da S. Lino, J. Loureiro, M.A.P. Lima, F.J. da Paixão Filho, Chem. Phys. 246, 275 (1999)

123. F.J. da Paixão Filho, M.A.P. Lima, V. McKoy, Phys. Rev. A 53, 1400 (1996)

124. R.B. Diniz, M.A.P. Lima, F.J. da Paixão Filho, J. Phys. B 32, L539 (1999)

125. M.H.F. Bettega, L.G. Ferreira, M.A.P. Lima, Phys. Rev. A 57, 4987 (1998)

126. A.P.P. Natalense, C.S. Sartori, L.G. Ferreira, M.A.P. Lima, Phys. Rev. A 54, 5435 (1996)

127. A.P.P. Natalense, L.G. Ferreira, M.A.P. Lima, Phys. Rev. Lett. 81, 3832 (1998)

128. R.F. da Costa, F.J. da Paixão, M.A.P. Lima, J. Phys. B 38, 4363 (2005)

129. R.F. da Costa, M.A.P. Lima, Int. J. Quantum Chem. 106, $2664(2006)$

130. R.F. da Costa, M.A.P. Lima, Phys. Rev. A 75022705 (2007)

131. R.F. da Costa, M.H.F. Bettega, M.A.P. Lima, Phys. Rev. A 77, 012717 (2008)

132. R.F. da Costa, M.H.F. Bettega, M.A.P. Lima, Phys. Rev. A 77, 042723 (2008)

133. R.F. da Costa, M.H.F. Bettega, M.A.P. Lima, M.C.A. Lopes, L. Hargreaves, G. Serna, M.A. Khakoo, Phys. Rev. A 85, 062706 (2012)

134. M.A. Khakoo, J. Muse, K. Ralphs, R.F. da Costa, M.H.F. Bettega, M.A.P. Lima, Phys. Rev. A 81, 062716 (2010)

135. K. Regeta, M. Allan, Phys. Rev. A 91, 012707 (2015)

136. J.B. Maljkovic, F. Blanco, R. Curik, G. García, B.P. Marinkovic, A.R. Milosavljevic, J. Chem. Phys. 137, 064312 (2012)

137. R.F.C. Neves, D.B. Jones, M.C.A. Lopes, K.L. Nixon, G.B. da Silva, H.V. Duque, E.M. de Oliveira, R.F. da Costa, M.T. do N. Varella, M.H.F. Bettega, M.A.P. Lima, K. Ratnavelu, G. García, M.J. Brunger, J. Chem. Phys. 142, 104305 (2015)
138. R.F. da Costa, M.H.F. Bettega, M.T. do N. Varella, E.M. de Oliveira, M.A.P. Lima, Phys. Rev. A 90, 052707 (2014)

139. T.P.T. Do, K.L. Nixon, M. Fuss, G. García, F. Blanco, M.J. Brunger, J. Chem. Phys. 136, 184313 (2012)

140. M. Allan, C. Winstead, V. McKoy, Phys. Rev. A 77, 042715 (2008)

141. H. Cho, R.J. Gulley, K. Sunohara, M. Kitajima, L.J. Uhlmann, H. Tanaka, S.J. Buckman, J. Phys. B 34, 1019 (2001)

142. G.L.C. de Souza, A.S. dos Santos, R.R. Lucchese, L.E. Machado, L.M. Brescansin, H.V. Manini, I. Iga, M.-T. Lee, Chem. Phys. 393, 19 (2012)

143. B. Mapstone, W.R. Newell, J. Phys. B 25, 491 (1992)

144. R. Panajotovic, M. Kitajima, H. Tanaka, M. Jelisavcic, J. Lower, L. Campbell, M.J. Brunger, S.J. Buckman, J. Phys. B 36, 1615 (2003)

145. M. Kitajima, Y. Sakamoto, R.J. Gulley, M. Hoshino, J.C. Gibson, H. Tanaka, S.J. Buckman, J. Phys. B 33, 1687 (2000)

146. M.A. Khakoo, K. Keane, C. Campbell, N. Guzman, K. Hazlett, J. Phys. B 40, 3601 (2007)

147. G. Staszewska, D.W. Schwenke, D. Thirumalai, D.G. Truhlar, J. Phys. B 16, L281 (1983)

148. G. Staszewska, D.W. Schwenke, D. Thirumalai, D.G. Truhlar, Phys. Rev. A 28, 2740 (1983)

149. G. Staszewska, D.W. Schwenke, D.G. Truhlar, Phys. Rev. A 29, 3078 (1984)

150. F. Blanco, G. García, Phys. Lett. A 255, 147 (1999)

151. F. Blanco, G. García, Phys. Lett. A 295, 178 (2002)

152. M.-T. Lee, I. Iga, L.E. Machado, L.M. Brescansin, E.A. y Castro, I.P. Sanches, G.L.C. de Souza, J. Electron. Spectrosc. Relat. Phenom. 155, 14 (2007)

153. G.L.C. de Souza, A.S. dos Santos, R.R. Lucchese, L.E. Machado, L.M. Brescansin, H.V. Manini, I. Iga, M.-T. Lee, Chem. Phys. 393, 19 (2012)

Open Access This is an open access article distributed under the terms of the Creative Commons Attribution License (http://creativecommons.org/licenses/by/4.0), which permits unrestricted use, distribution, and reproduction in any medium, provided the original work is properly cited. 